\title{
The Role of the Employer in Supporting Work Participation of Workers with Disabilities: A Systematic Literature Review Using an Interdisciplinary Approach
}

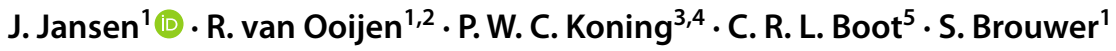

Accepted: 16 April 2021 / Published online: 12 May 2021

(c) The Author(s) 2021

\begin{abstract}
Purpose There is growing awareness that the employer plays an important role in preventing early labor market exit of workers with poor health. This systematic review aims to explore the employer characteristics associated with work participation of workers with disabilities. An interdisciplinary approach was used to capture relevant characteristics at all organizational levels. Methods To identify relevant longitudinal observational studies, a systematic literature search was conducted in PubMed, Web of Science, PsycINFO and EconLit. Three key concepts were central to the search: (a) employer characteristics, (b) work participation, including continued employment, return to work and long-term work disability, and (c) chronic diseases. Results The search strategy resulted in 4456 articles. In total 50 articles met the inclusion criteria. We found 14 determinants clustered in four domains: work accommodations, social support, organizational culture and company characteristics. On supervisor level, strong evidence was found for an association between work accommodations and continued employment and return to work. Moderate evidence was found for an association between social support and return to work. On higher organizational level, weak evidence was found for an association between organizational culture and return to work. Inconsistent evidence was found for an association between company characteristics and the three work outcomes. Conclusions Our review indicates the importance of different employer efforts for work participation of workers with disabilities. Workplace programs aimed at facilitating work accommodations and supervisor support can contribute to the prevention of early labor market exit of workers with poor health. Further research is needed on the influence of organizational culture and company characteristics on work participation.
\end{abstract}

Keywords People with disabilities $\cdot$ Return to work $\cdot$ Employment $\cdot$ Social support $\cdot$ Workplace

\section{Introduction}

J. Jansen

j.jansen@umcg.nl

1 Department of Health Sciences, Community and Occupational Medicine, University of Groningen, University Medical Center Groningen, Groningen, The Netherlands

2 Department of Economics, Econometrics and Finance, University of Groningen, Groningen, The Netherlands

3 Department of Economics, VU University Amsterdam, Amsterdam, The Netherlands

4 Department of Economics, Leiden University, Leiden, The Netherlands

5 Department of Public and Occupational Health, Amsterdam UMC, VU University Amsterdam, Amsterdam Public Health Research Institute, Amsterdam, The Netherlands
Several OECD countries reformed their disability programs over the past decades to foster labor market integration of people who face challenges staying or re-entering the workforce due to illness or disabilities [1]. These reforms primarily focused on the reintegration of workers with disabilities into employment; recognizing that many of them only have partially reduced work capacity and could therefore continue working if adequately supported by their employer [1-3]. Following these reforms the employment rates of people with disabilities has increased over the years [1,4].This suggests that employment outcomes of people with disabilities are not only affected by their health conditions but also by their work environment [5].

As a result, there is growing awareness that the employers' organizational context plays an important role in 
preventing early labor market exit of workers with poor health. The organizational context is defined as the characteristics of a workplace, including the social, physical and organizational structure of a company [6]. As such, both the employers' disability management policies and practices and the social interaction between employers and employees may influence job retention of employees with disabilities [7]. An employer can, for instance, support employees with disabilities by offering workplace accommodations with the aim to improve job functioning, facilitate faster return to work, and remove job related barriers [8].

In occupational health care, several studies have been published about employer-related determinants and intervention strategies that improve labor market participation of workers with disabling health conditions. These studies in particular focus on workers with musculoskeletal disorders [9-12], mental health conditions [10, 13] and/or cancer [14, 15]. Besides company characteristics, supervisor support is often reported as an important employer-related determinant of return to work, however findings are mixed [9, 13, 14]. Employer-related intervention strategies in particular focus on workplace accommodations used by employers to recruit, hire, retain, and promote persons with physical disabilities, i.e. physical/technological modifications, accommodations to enhance workplace flexibility and worker autonomy and strategies to promote workplace inclusion and integration [16]. Rigorous evaluations of the effectiveness of these accommodations is not well-documented in peer reviewed literature yet $[10,16]$. Economic studies, on the other hand, often focus on the overall effectiveness of work accommodations regardless of the cause, across all types of health conditions, and frequently focus on the costs and benefits of different return-to-work programs, to learn what program works best. Another strength of the economics field is their use of largescale register data, adding knowledge to the field of occupational health. Each discipline and its corresponding research methods thus provides different insights about employer efforts and work participation of workers with disabilities, making them complementary to each other. As the topic of employer support for workers with disabilities is being investigated by different disciplines, an interdisciplinary approach is crucial to obtain a complete overview.

Moreover, to get a better insight into the role of employers in supporting workers with disabilities to continue their jobs it is important take into account the role of the employer at all organizational levels. Rather than only focusing on work accommodations, as was the focus of previous reviews [16], we strive to include a broader range of employer efforts by integrating the existing evidence from different disciplines. Such an interdisciplinary approach requires a comparison of different types of work disabilities and work participation outcomes, because different outcomes and types of work disabilities are considered relevant in different disciplines.
In addition, in contrast to other reviews we include longitudinal quantitative studies which allows us to summarize the evidence of the associations between prognostic factors at the employer level, and long-term work outcomes. Therefore, we will focus on three long-term work participation outcomes: return to work, continued employment and longterm disability. To date, such an integration of the existing evidence on prognostic factors at employer level from different disciplines has not been conducted.

Thus, this systematic review aims to explore the employer characteristics associated with work participation of workers with disabilities through an interdisciplinary approach including an occupational health, psychology and economic perspective.

\section{Method}

\section{Search Strategy}

We conducted an interdisciplinary search using four databases: Pubmed, PsycINFO, Web of Science and EconLit (inception of databases until 17 April 2018). Pubmed was selected for its coverage of health and medicine-focused journals. PsycINFO was selected for its coverage of journals with a focus on psychology. Web of Science was selected for its coverage of occupational health journals. EconLit was selected for its coverage of economic journals. The key concepts used in the search strategy were developed by the research team with the support of a university librarian with an expertise on making systematic review searches. Three key concepts were central to the search: (a) employer characteristics; (b) work participation; and (c) chronic diseases. Synonyms were identified for each concept, including keywords and phrases as well as database-specific subject headings (e.g. MeSH headings) (online supplementary text S1). The search terms were adapted to each database to best utilize the search functionality and controlled vocabularies unique to each of them.

\section{Selection of Studies}

Two independent reviewers ( $\mathrm{JJ}, \mathrm{RvO}$ ) performed the selection of the studies in three screening phases. In the first phase, articles were excluded based on titles and abstracts. The systematic reviews application Rayyan was used for the initial screening of titles and abstracts [17]. All peerreviewed journal articles were screened according to predefined criteria by the research team: (i) the study population consisted of workers with a chronic disease; (ii) the subjects were aged 18-67 years (i.e., working age population); (iii) the study used a longitudinal quantitative study design; (iv) the study examined continued employment, return to work 
after $>3$ months of sickness absence, or long-term sickness absence (>3 months) as the outcome variable; (v) at least one of the independent variables contains employer characteristics, including the role of professionals if they interact with the employer; and (vi) the article was written in English. As a consequence these articles are mostly from western countries. In the second phase, the reviewers selected articles for final inclusion based on full-text appraisal. Studies were excluded when both reviewers considered that these did not fulfil the inclusion criteria. Disagreements regarding inclusion were resolved by consensus. If no consensus was reached or in case of doubt, the article was screened by the other authors and discussed to reach consensus. In the third phase, references of included articles were checked for additional relevant articles and we checked for additional recently published articles from the field of economics because of its relatively lengthy publishing process.

\section{Data Extraction}

Two reviewers (JJ, RvO) independently extracted the following characteristics from the included studies: study design, country of the study, scientific discipline, follow-up time, general description of subjects including age and gender, work disability type, outcome measures, employer characteristics and effect sign and size.

\section{Assessment of Quality}

Two reviewers (JJ, RvO) independently assessed the methodological quality of the included studies using nine items $[18,19]$. This quality checklist is suitable for assessment of longitudinal observational studies [19]. Table 1 shows the standardized checklist for the quality assessment. Each item was scored positive (+) or negative (-). A negative score was seen as potential bias. The grading of each item was discussed between the reviewers to reach consensus. Based on the nine criteria, the studies were classified as being of high quality when meeting $\geq 8$ criteria, medium quality when meeting 6-7 criteria, and low quality when meeting $<6$ criteria [11].

\section{Evidence Synthesis}

A descriptive analysis was undertaken to synthesize the data, which consisted of four stages: grouping, clustering, transforming data and tabulation. Determinants were listed in a stepwise procedure per outcome measure: continued employment, return to work and long-term disability. First, an overview of all determinants that were studied in relation to the work outcomes was created. Determinants referring to the same concept were merged together. For example, the data extraction revealed different aspects of organizational culture, these were merged for evidence grading. Next, determinants were grouped into the following domains: work accommodations, supervisor support, organizational culture and company characteristics. Thirdly, we harmonized the direction of effect sizes. Lastly, we summarized for each domain: (i) the total number of studies reporting on the factor, (i) the number of studies of low, moderate and high quality reporting on the factor, (iii) the scientific disciplines, and (iv) disability types.

\section{Evidence Grading}

The level of evidence of the determinants was graded by using the rating system mentioned by de Croon et al. [9]. Ten different evidence levels were determined based on the number of studies and the directions of the effect size. The different evidence grading steps are shown in Fig. 1. Mixed results among the studies with a given outcome does not mean no effect; it means a mixture of negative and positive associations. The level of evidence was established per determinant.

Table 1 Checklist of methodological quality [18]

\begin{tabular}{ll}
\hline Potential biases & Quality assessment criteria \\
\hline Objective & 1. Positive if a clearly stated objective is described \\
Study population & 2. Positive if the main features of the study population are clearly described \\
& 3. Positive if the inclusion and exclusion criteria are clearly described \\
Outcome & 4. Positive if outcome is register-based and if not register-based, the loss to follow up is limited $(<20 \%)$ \\
5eterminant & 6. Positive if a clear definition of employment outcome is given \\
Analysis & 7. Positive if age (if possible), gender (if possible), education and income are taken into account as confounders \\
& 8. Positive if appropriate statistical model is used to evaluate data \\
9. Positive if effect size of variables was presented or p-value 0.05 was shown or can be calculated
\end{tabular}




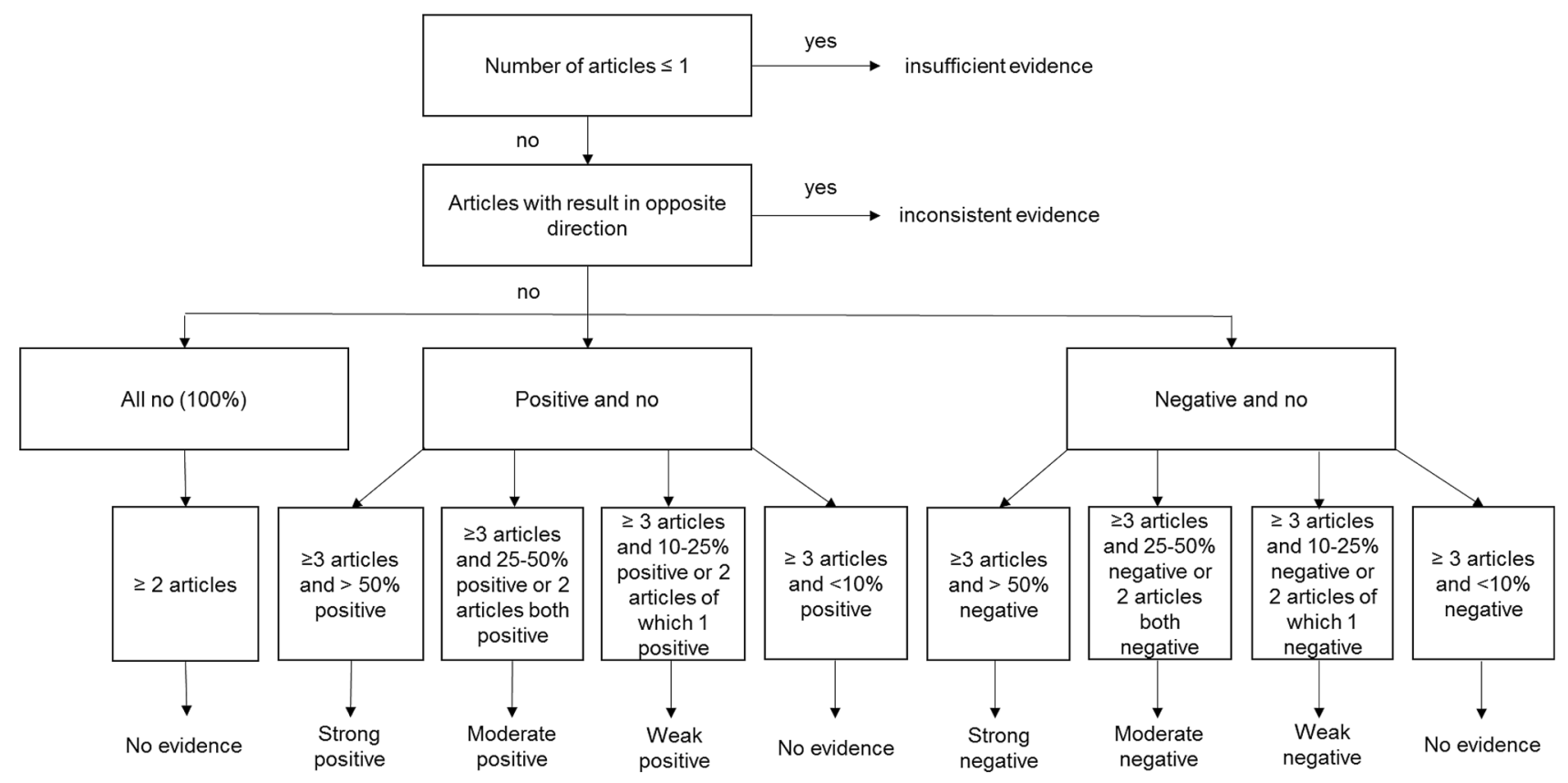

Fig. 1 Evidence grading

\section{Results}

\section{Selection of Studies}

The search strategy resulted in 4456 articles, of which 2817 were extracted from Pubmed, 2734 from Web of Science, 1140 from PsycINFO, and 37 from EconLit. After screening on titles and abstracts by the two reviewers, 4251 articles were excluded. A total of 205 articles were selected for further screening. Finally, 38 articles met all inclusion criteria. Further reference checking identified an additional 12 articles, resulting in 50 included articles on 52 individual studies. Figure 2 presents the flow diagram of the selection of studies.

\section{Study Characteristics}

The main characteristics of the included studies are presented in Table 2. Studies varied in work participation outcome measure, scientific disciplines and disability types. Of the 52 studies, 40 investigated determinants in relation to return to work outcomes, 11 studied determinants of continued employment and six studies used long-term disability as a work participation outcome. The economic discipline was represented in 15 studies; the medical discipline in 37 studies. Finally, 28 studies had a specific focus on one specific disability type: mental $(n=11)$, musculoskeletal $(n=7)$, cancer $(n=9)$, diabetes $(n=3)$, circulatory $(n=2)$ and nervous $(n=2)$. The other 20 studies had a broader focus, referred to as work-limiting health conditions. The effect sizes are reported in Table 2 in odds ratios (OR), hazard ratios (HR), rate ratios (RR), propensity score matching (PSM) and marginal effects (ME). The outcome column describes effect sizes of the association between the employer determinant and the outcome, measured at the indicated follow-up period.

\section{Quality Assessment}

The results of the quality assessment are presented in Table 3. In total, 39 out of 50 articles (78\%) were graded to be of high quality, whereas the other 11 articles (22\%) were graded as medium quality. No low quality articles were found.

\section{Employer Determinants}

In total, we found 14 determinants that could be clustered in the following four domains: work accommodations, social support, organizational culture and company characteristics (see Table 4).

\section{Work Accommodations}

Work accommodation, defined in studies as having an accommodating employer or offered accommodations, was found to be related to continued employment [20-24] and faster return to work [25-29]. Moderate evidence was found for this determinant related to reduced long-term disability $[21,30,31]$. 


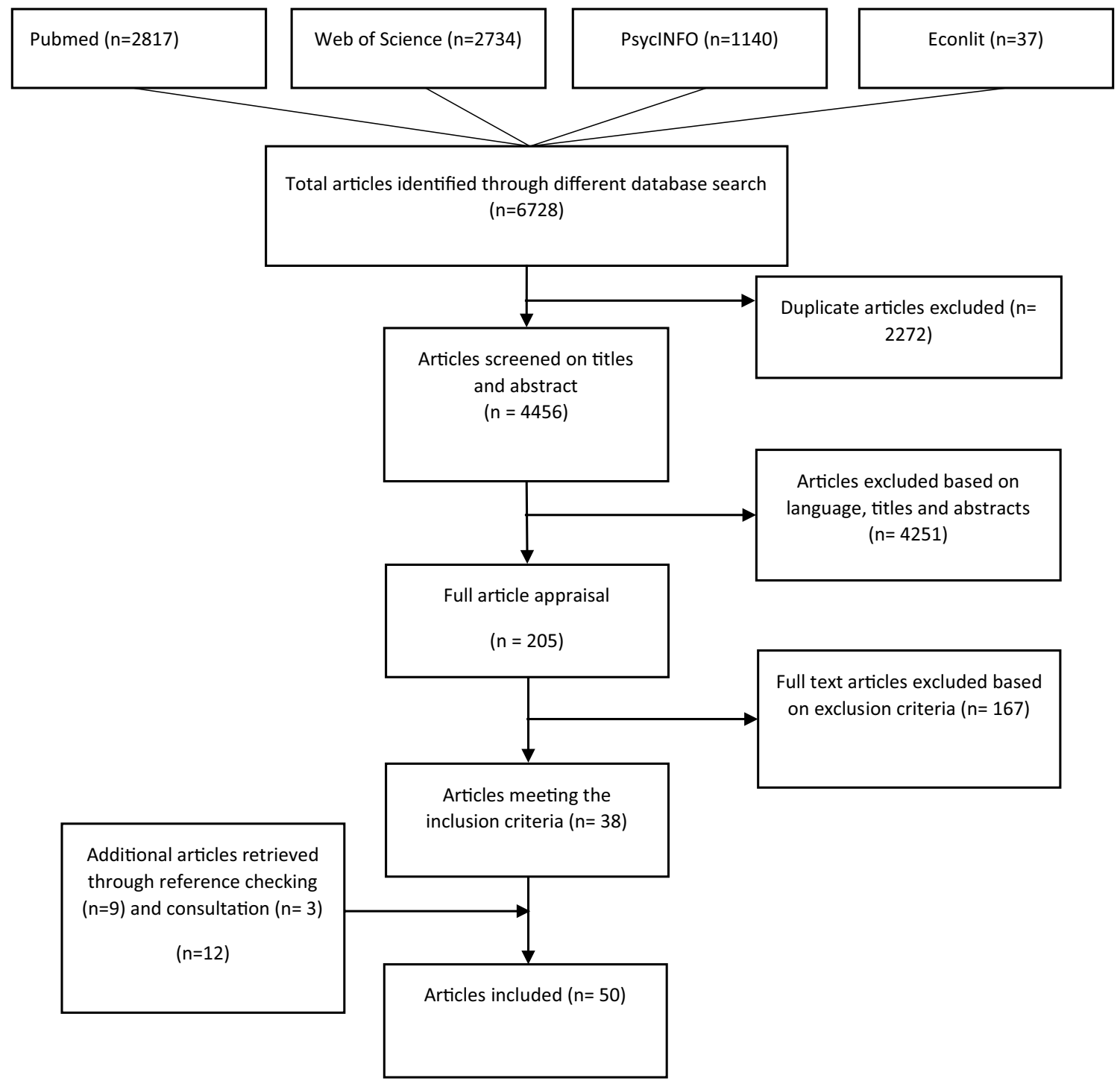

Fig. 2 Flow diagram of the selection of studies

Nine different types of work accommodations were studied: work change, employer change, work-time change, workplace interventions, professional assistance at the workplace, professional assistance outside the workplace, graded return to work, equipment assistance, and employer provided health/disability insurance. There was moderate evidence that work change, defined as change in job tasks and change in work, was positively associated with continued employment [21-23, 32]. Change in work time and flexibility in time scheduling was strongly positively associated with return to work [28, 33, 34]. There was less evidence pointing at effects of change in work time on continued employment [21-23] and employer change [22, 43]. Workplace programs on guidance and support such as vocational work training, case management interviews and occupational health services was strongly positively associated with return to work [26, 33, 35-38]. In addition, we found weak evidence for a positive association between graded return to work programs and return to work [39-42], and a weak positive association between equipment assistance and continued employment [21-23]. Strong evidence was found between equipment assistance and return to work [27, 28, 33]. For return to work, we found inconsistent evidence for the following determinants: work change $[28,33,35]$ and professional assistance outside the workplace [26, 27, 40].

For some determinants and outcomes, we did not find sufficient studies to assess the evidence. For continued employment, this was the case for the following determinants: graded return to work [42], professional assistance at work [23] and professional assistance outside the workplace [23]. For return to work, this concerns the determinant professional assistance at the workplace [27]. For long-term 


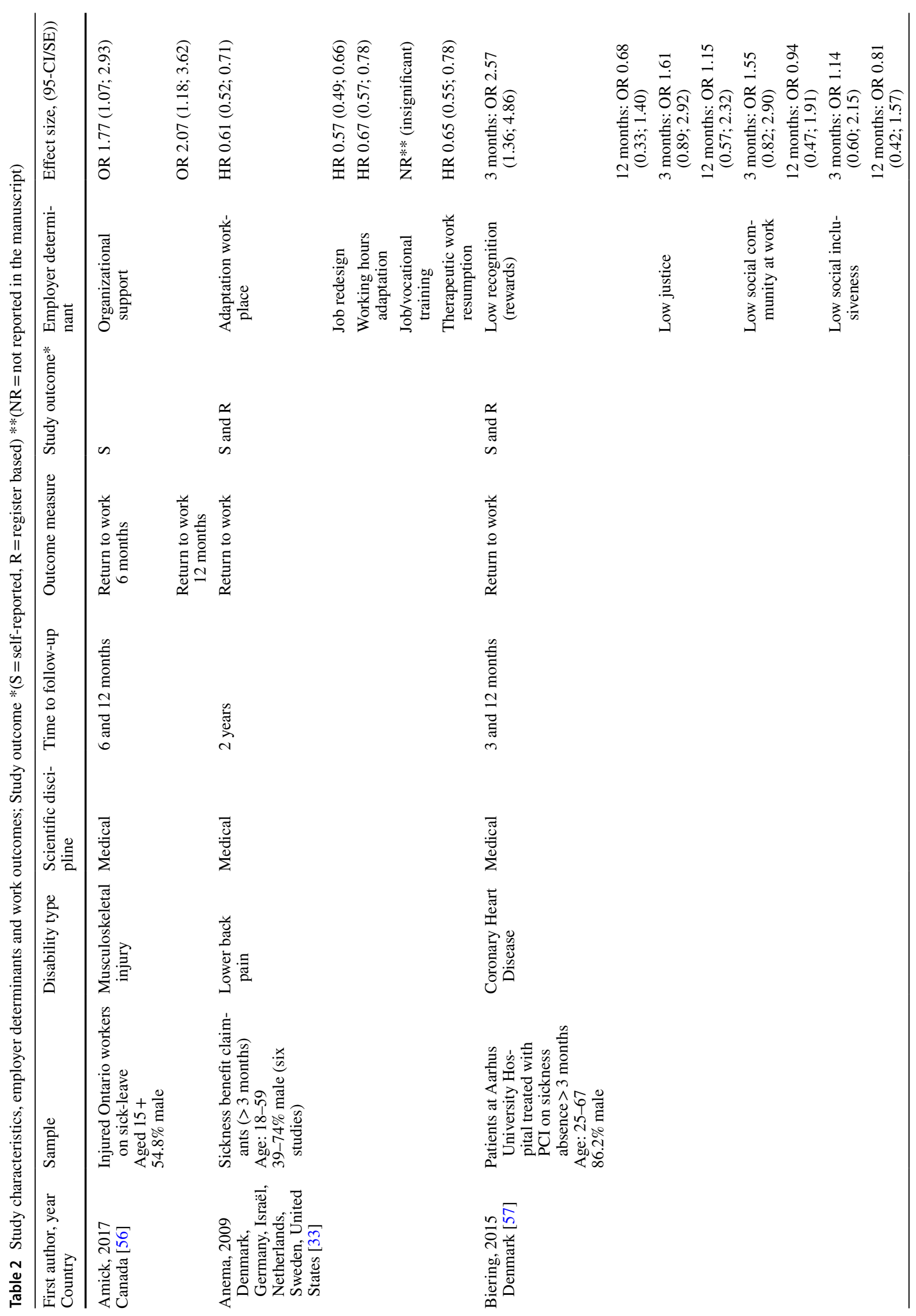




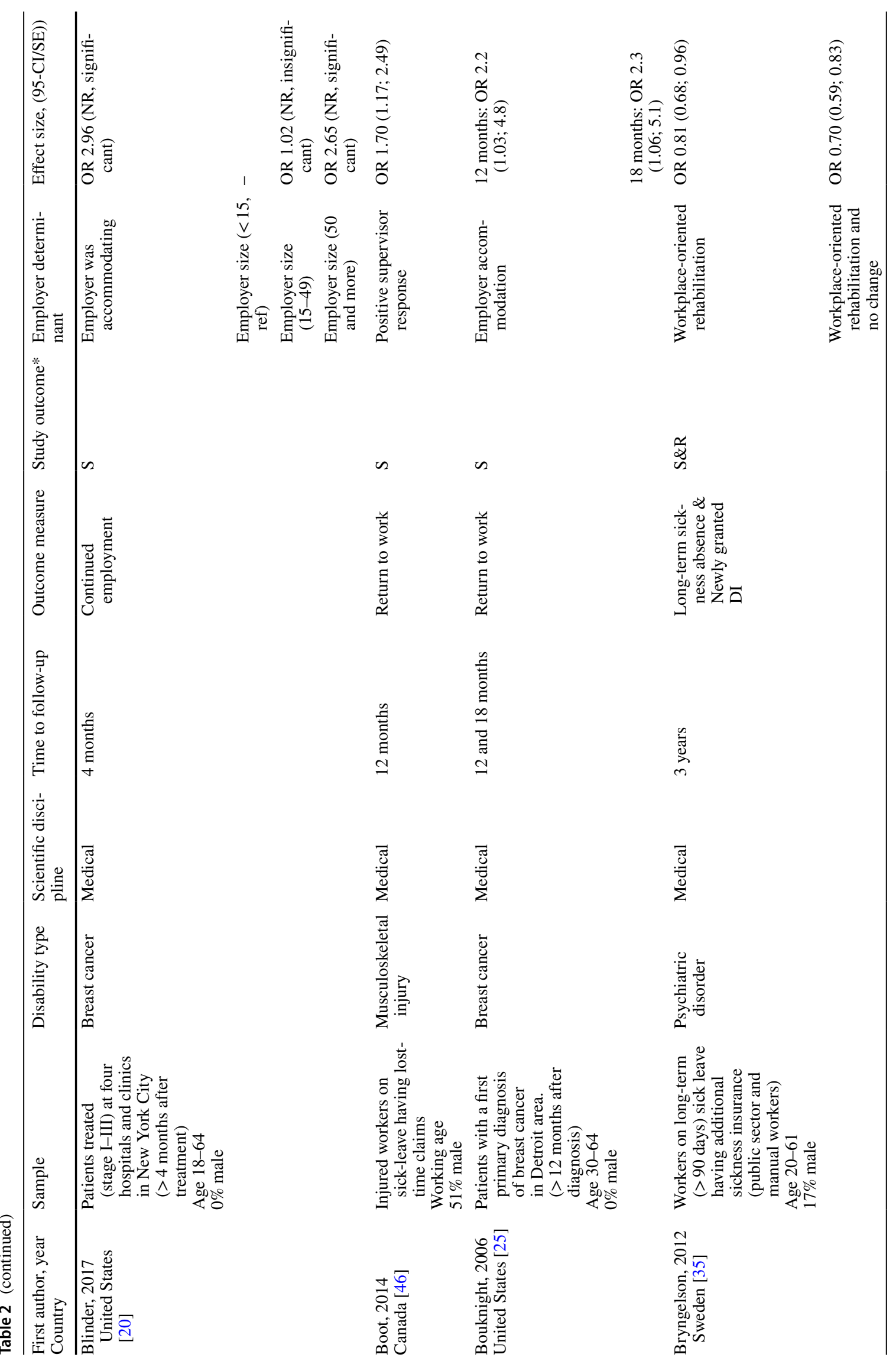




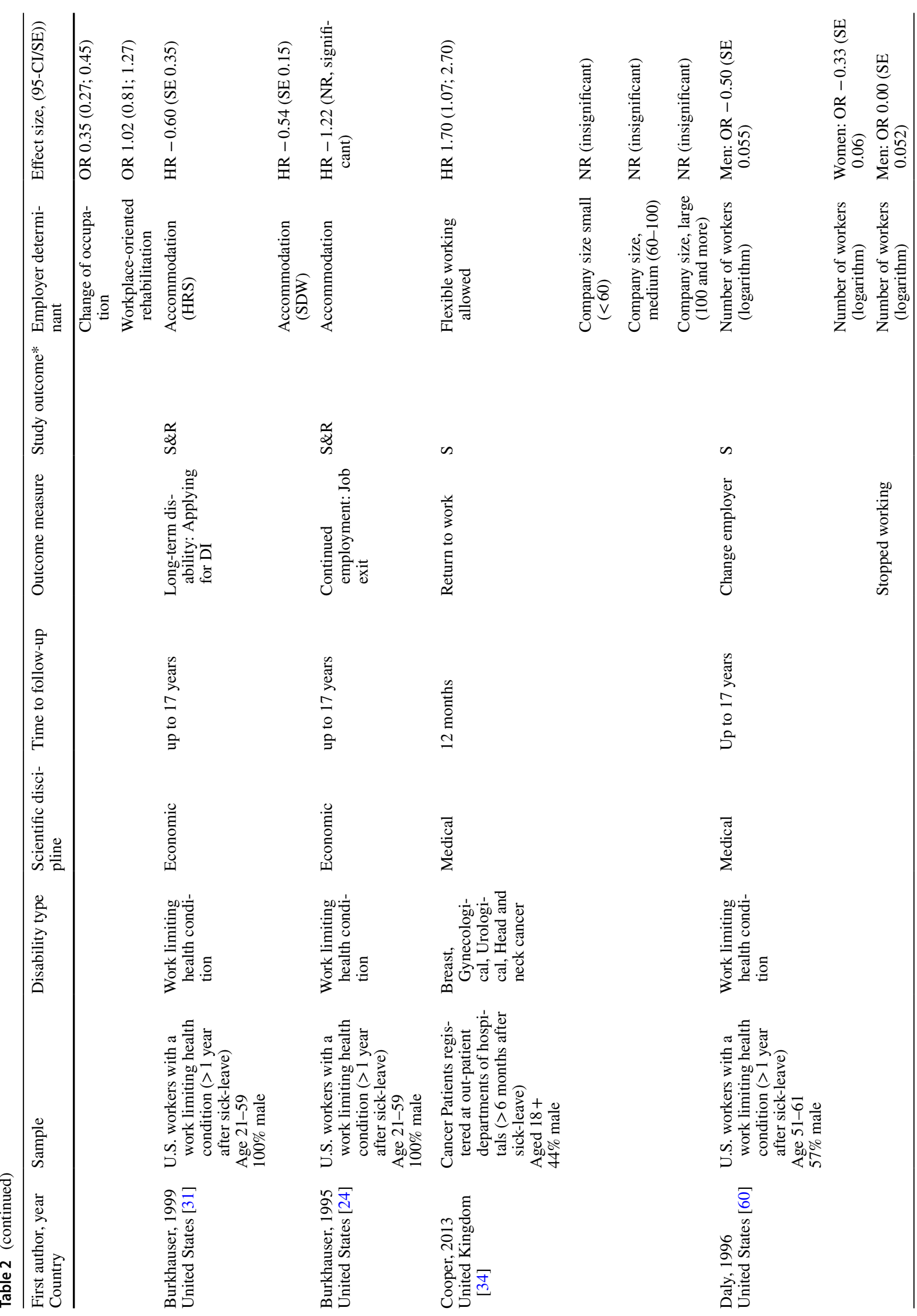




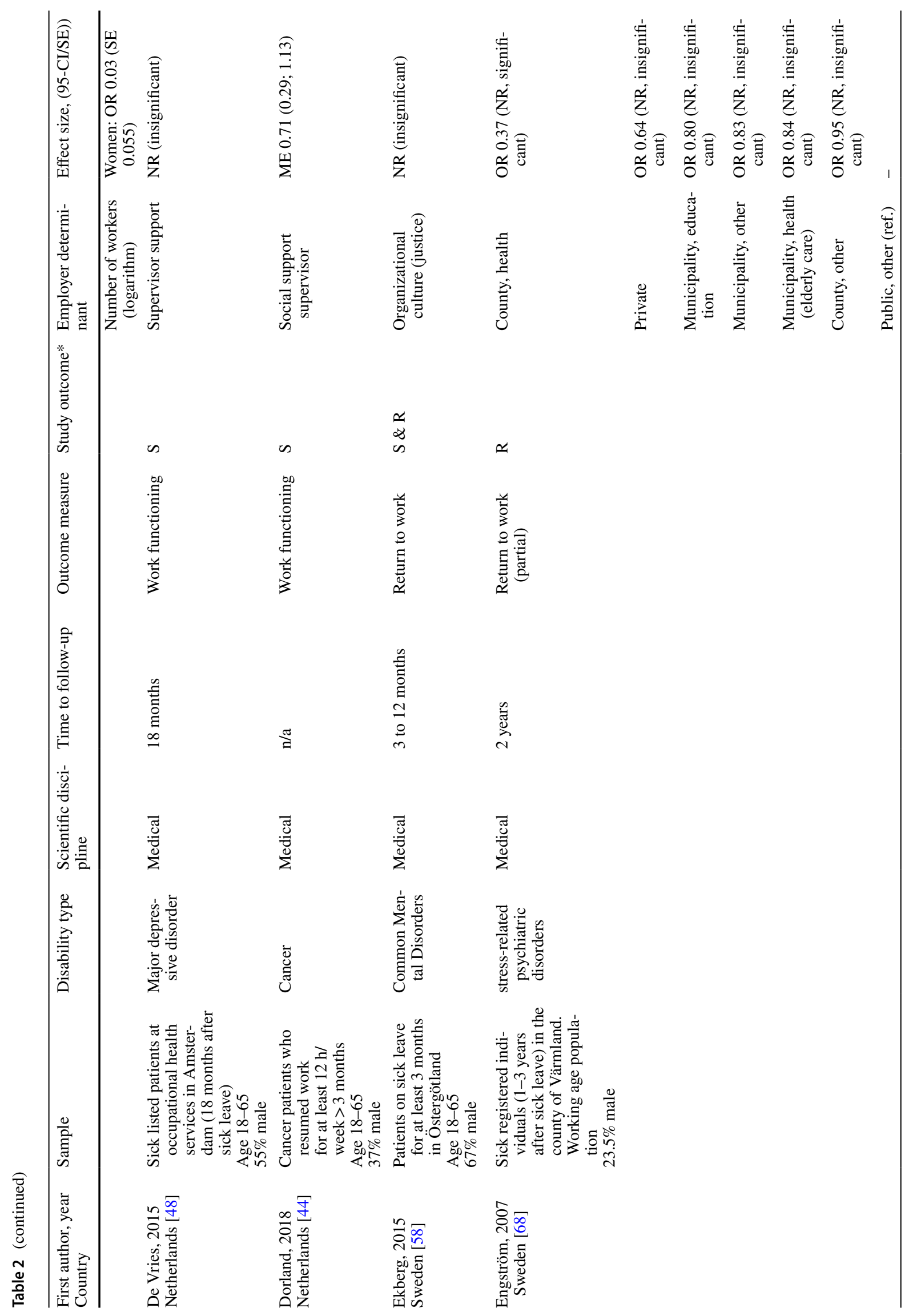




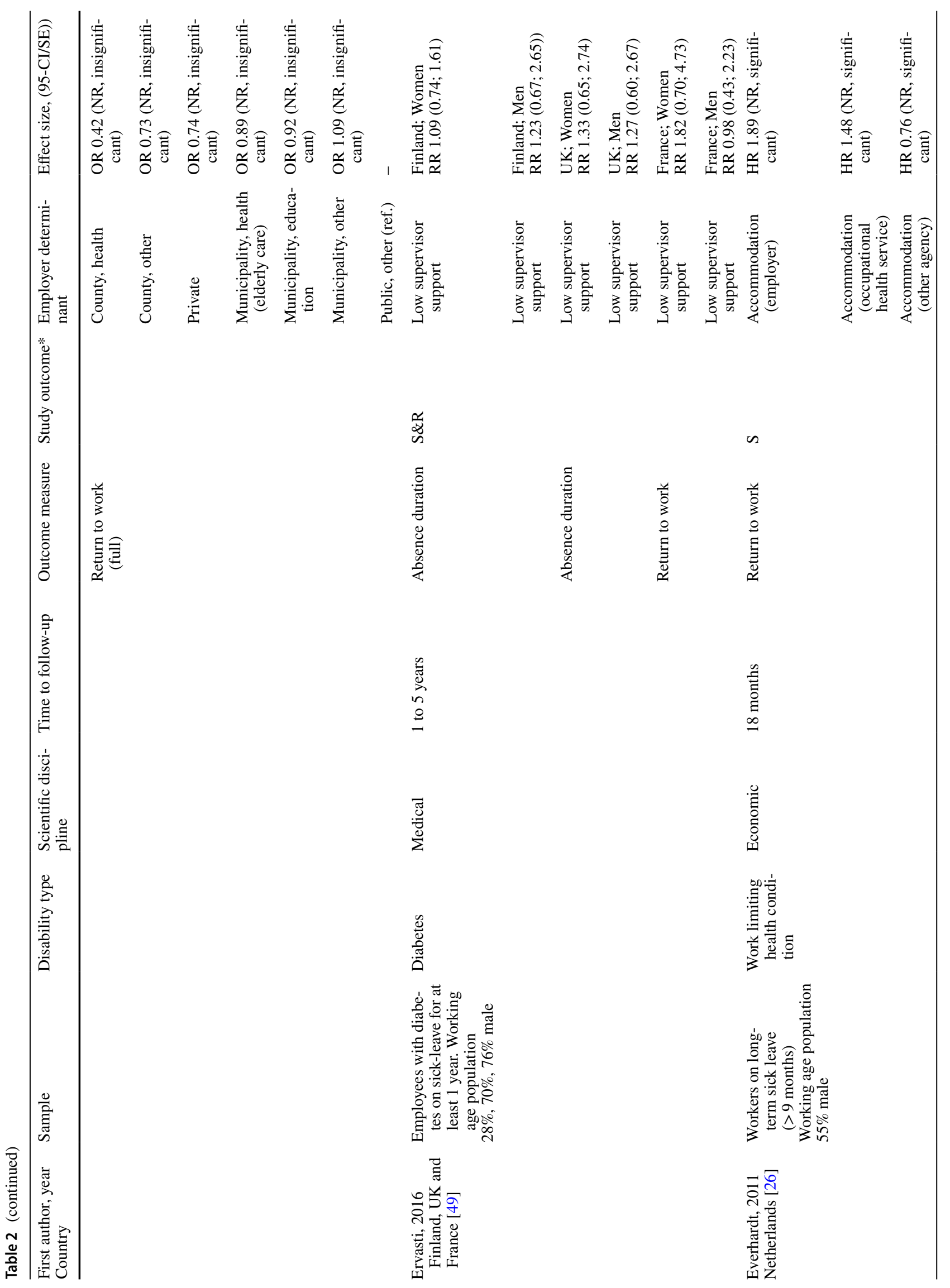




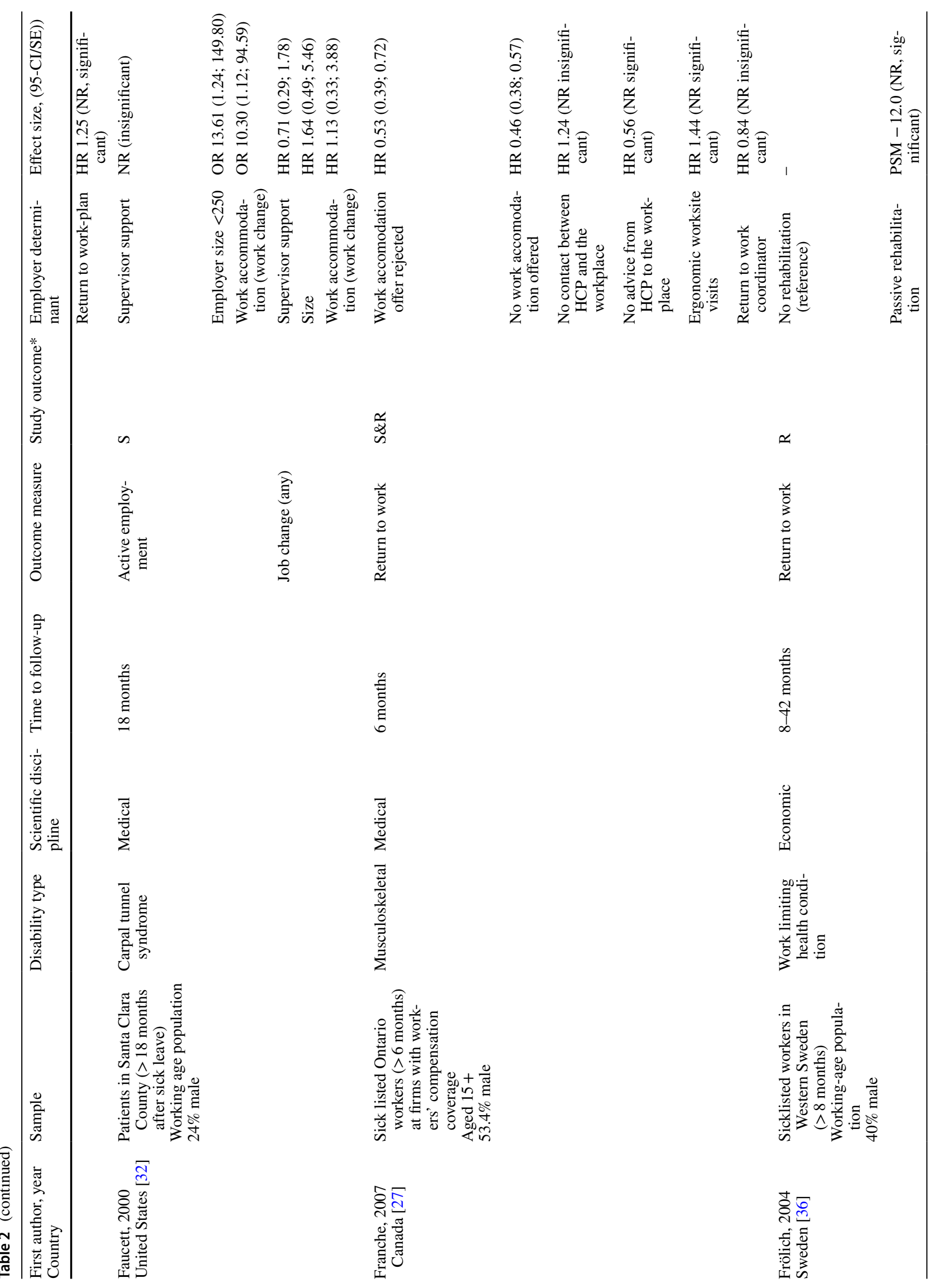




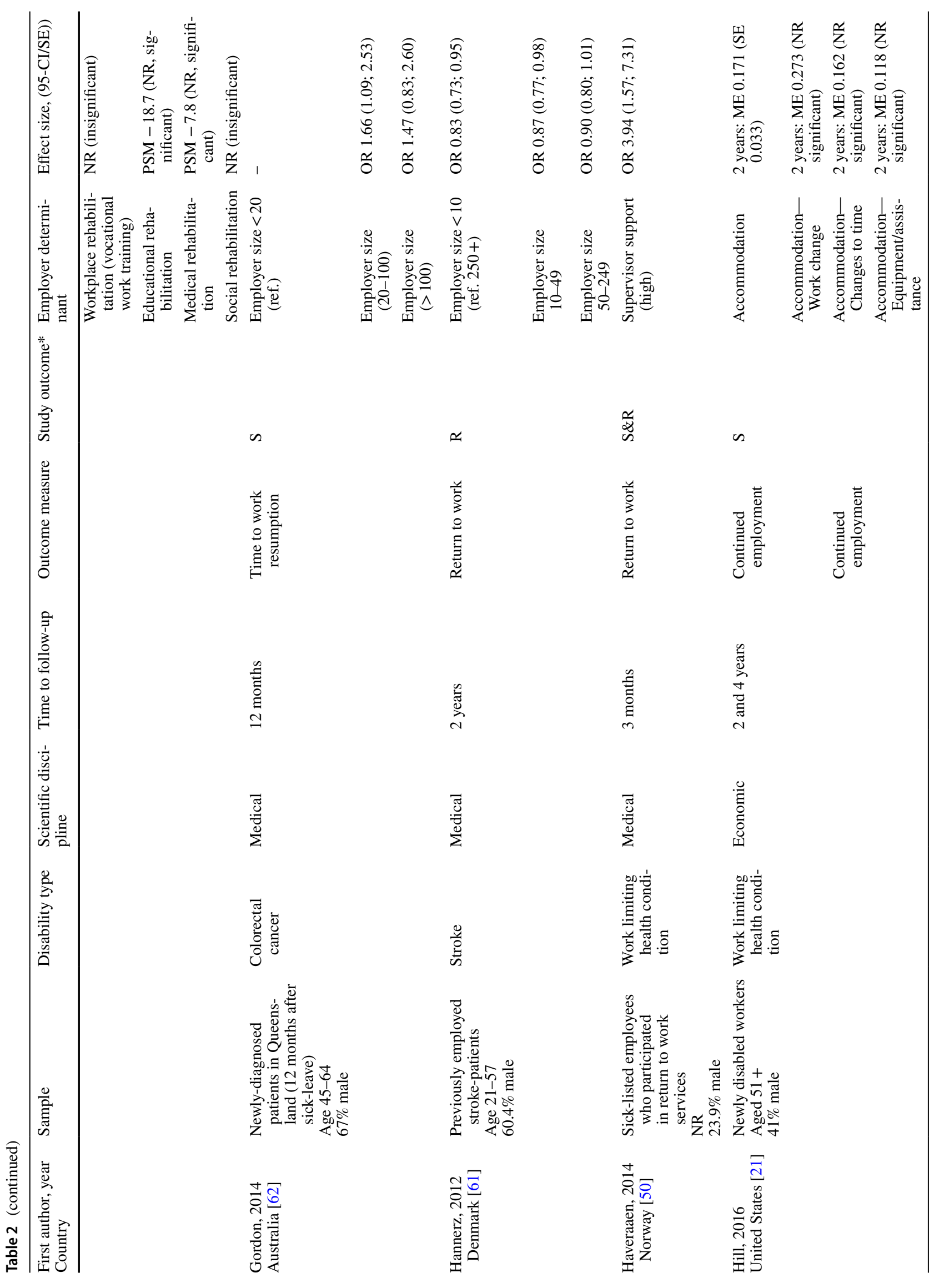




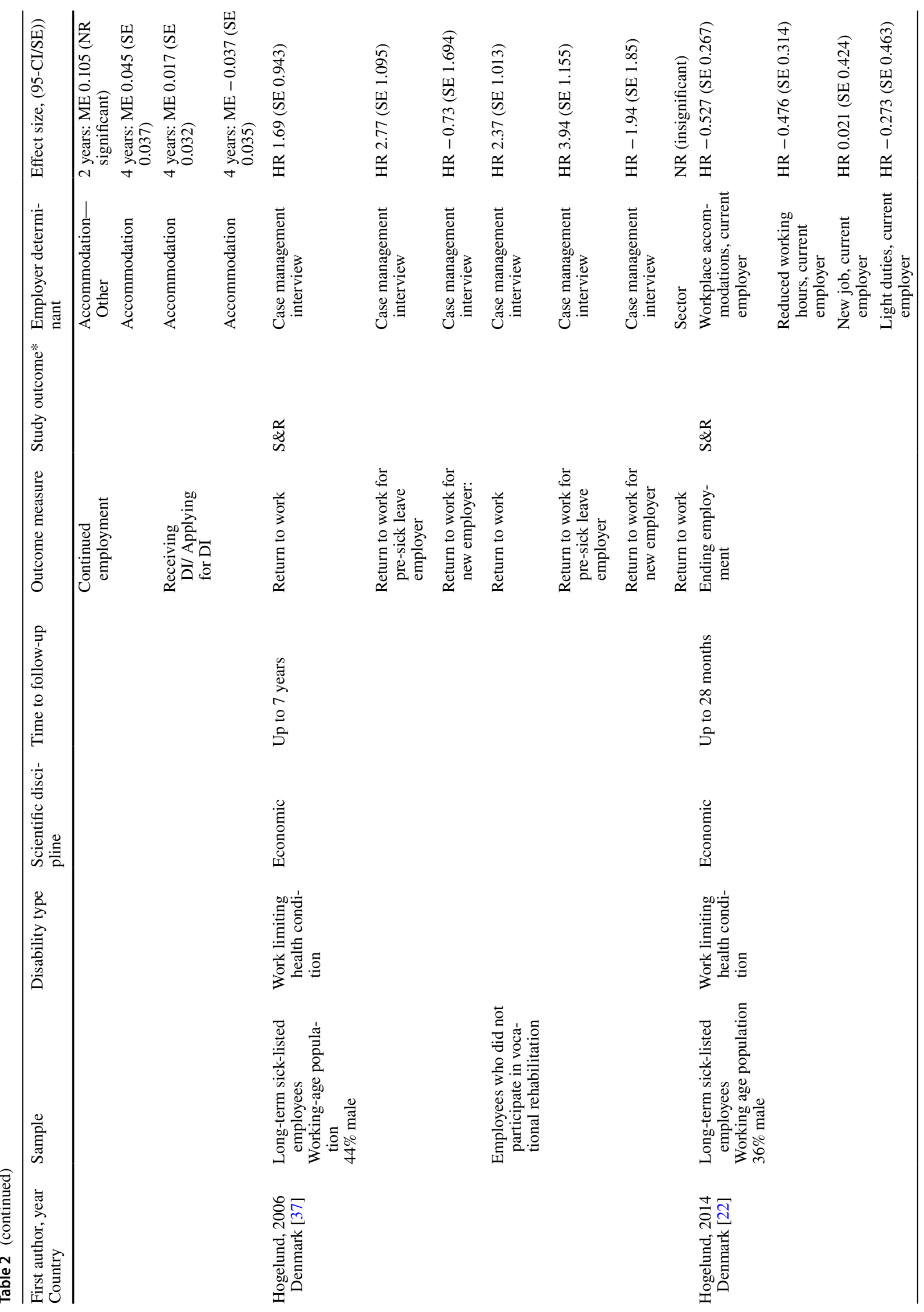




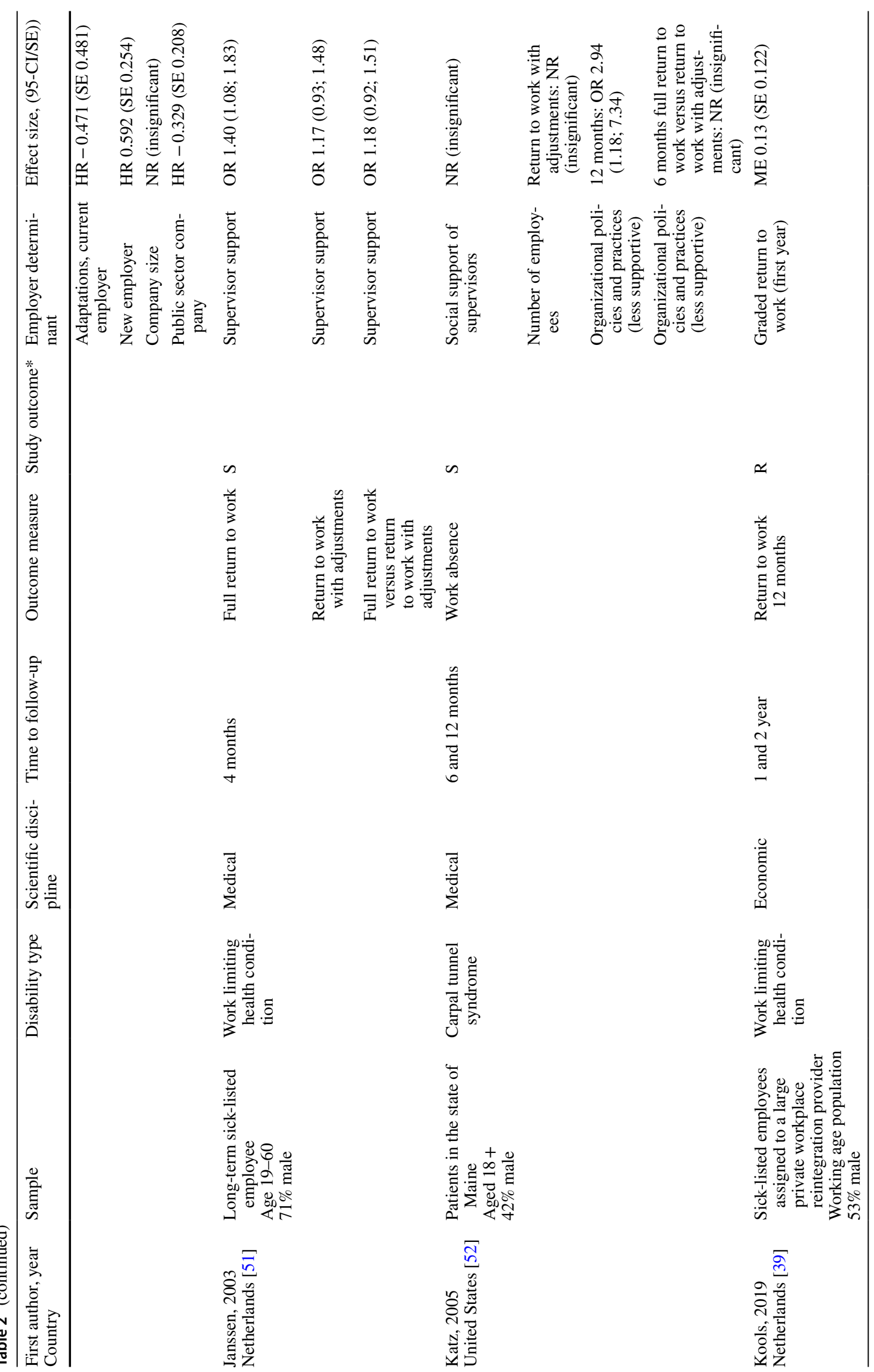




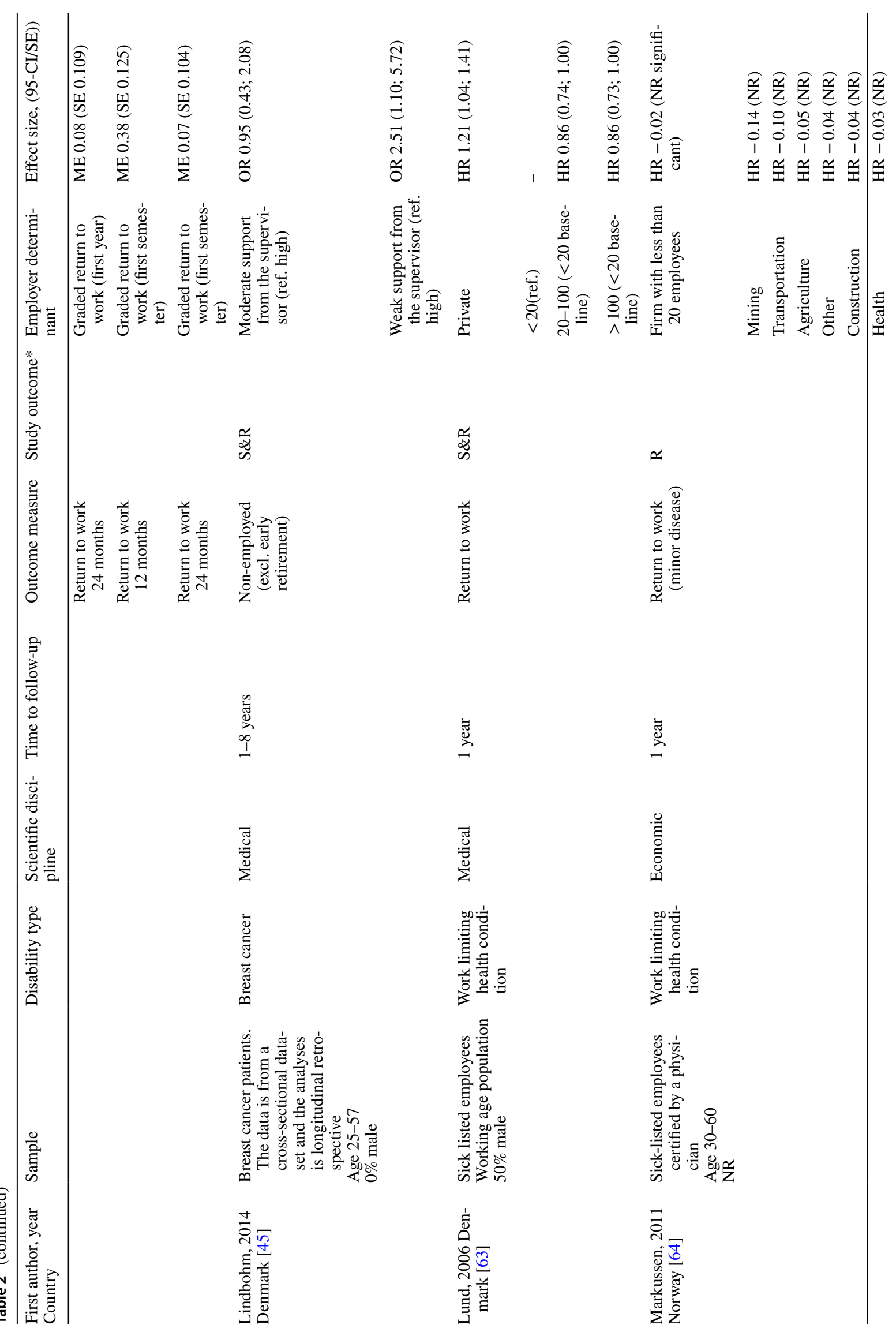




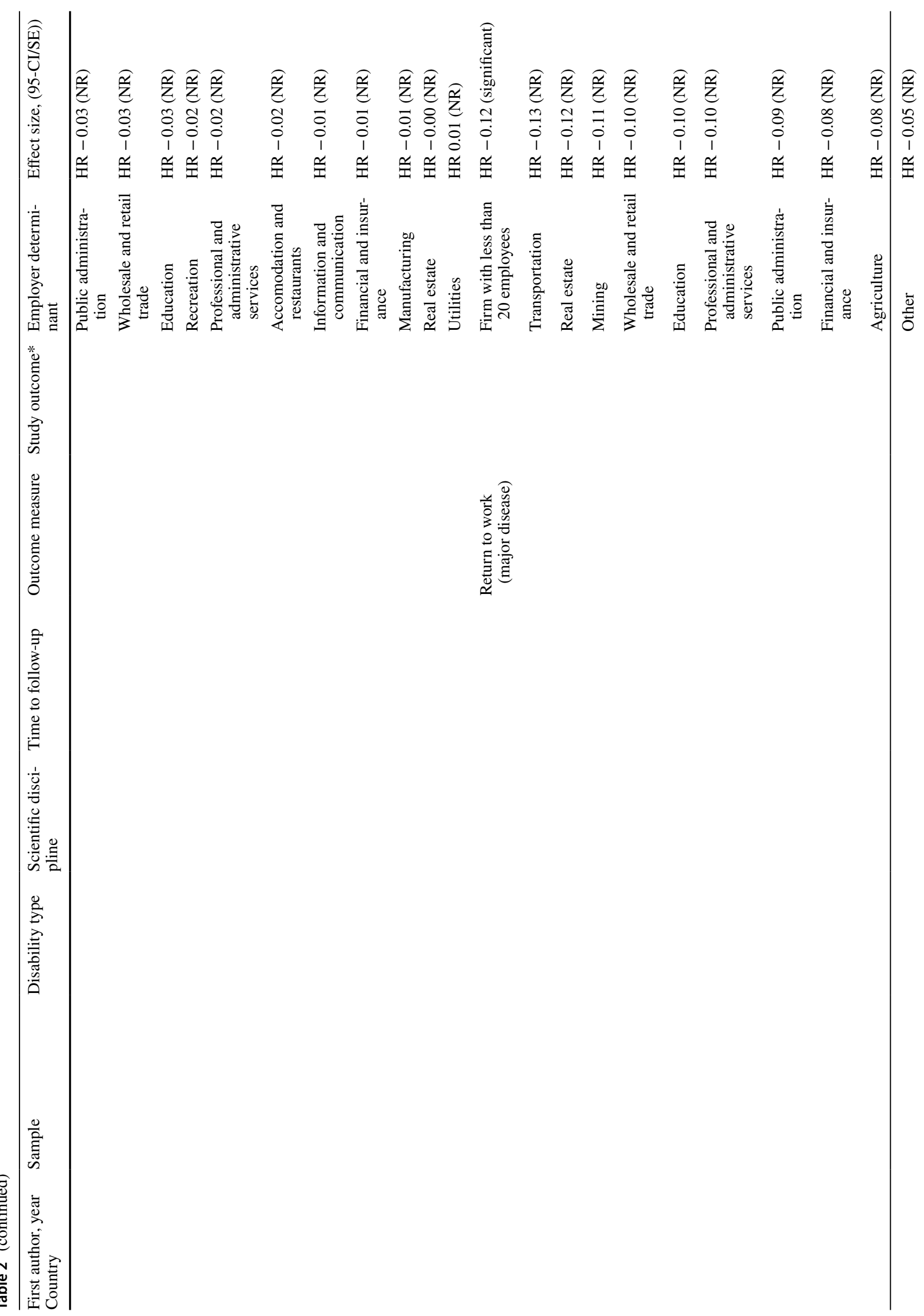




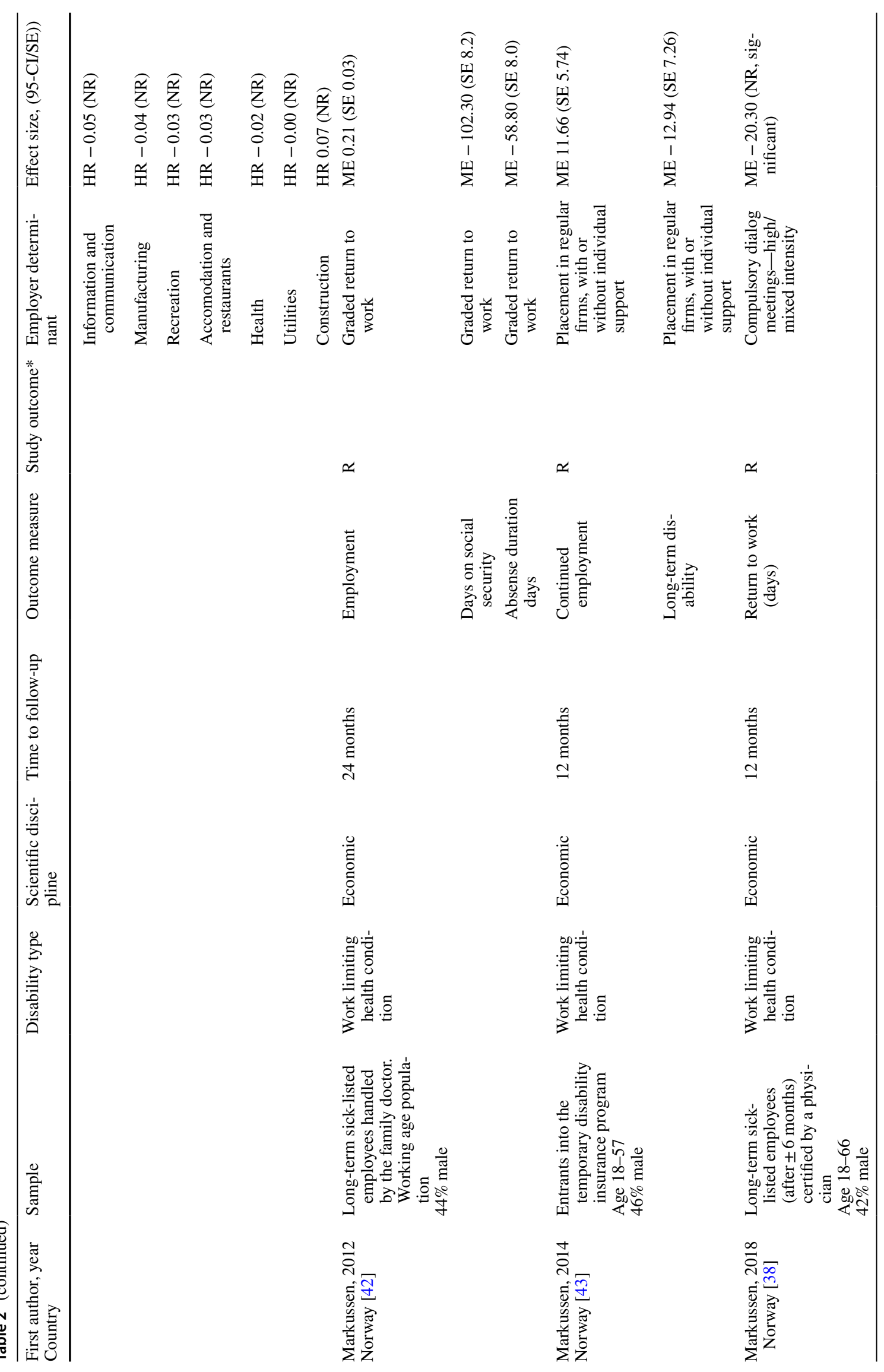




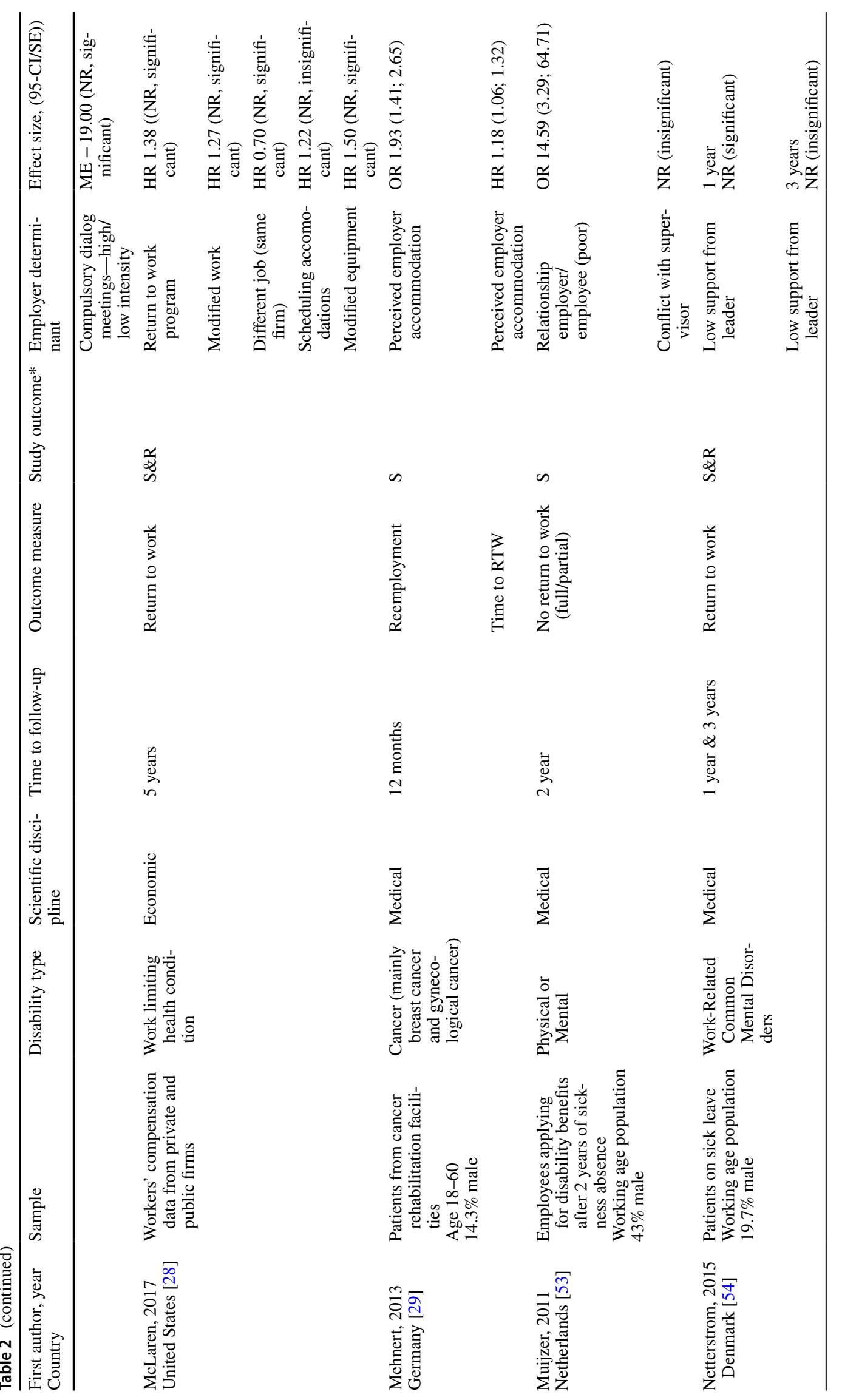




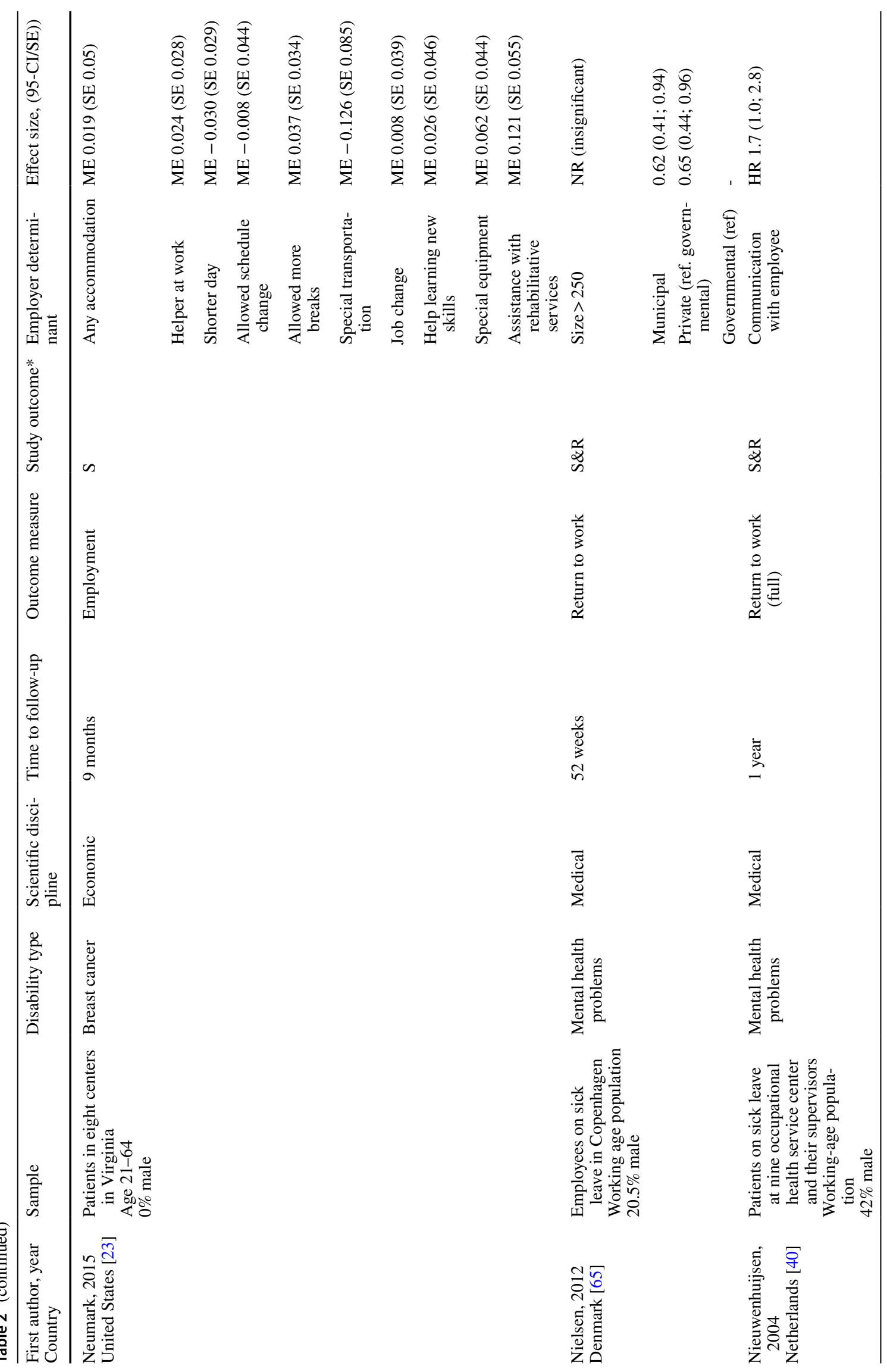




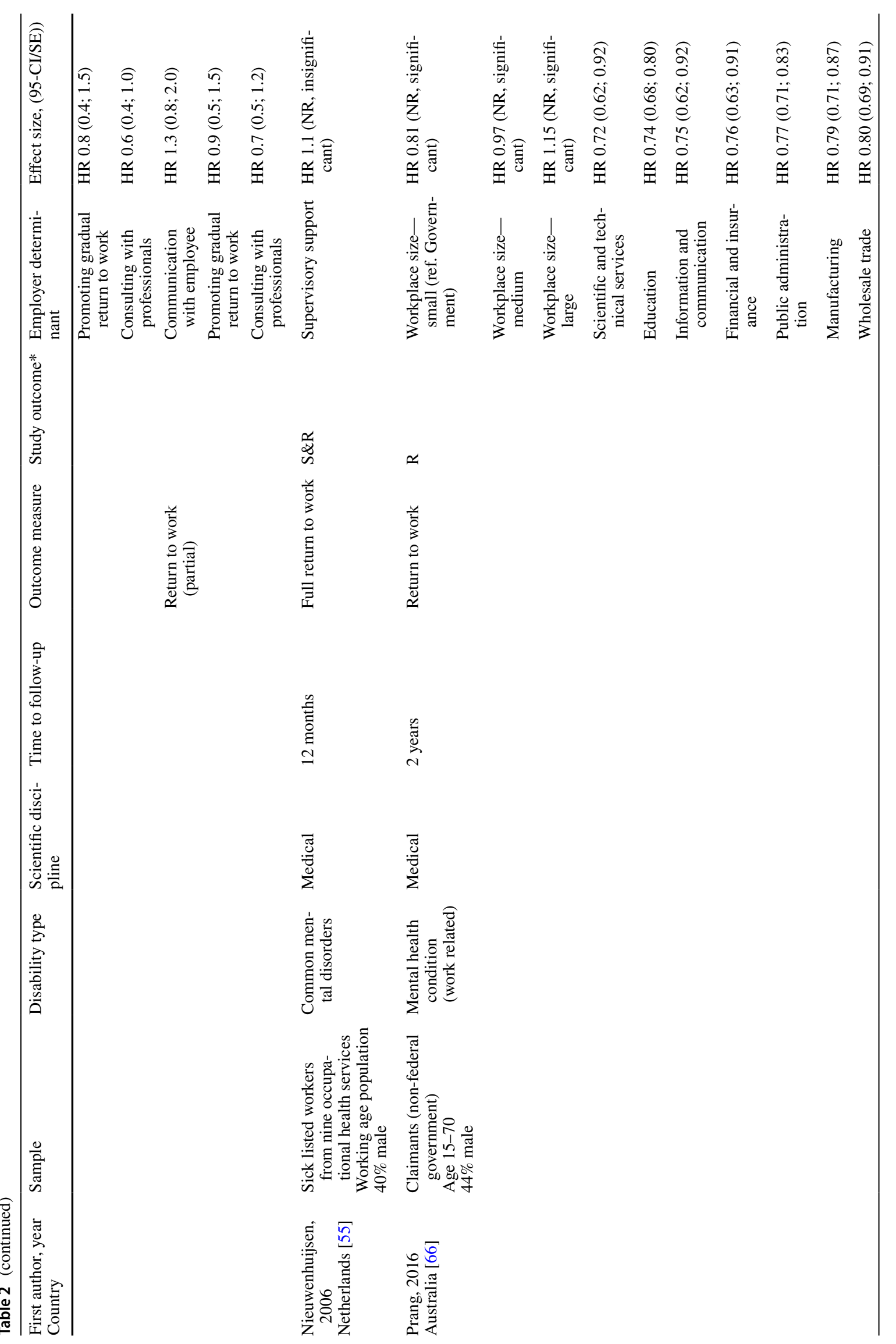




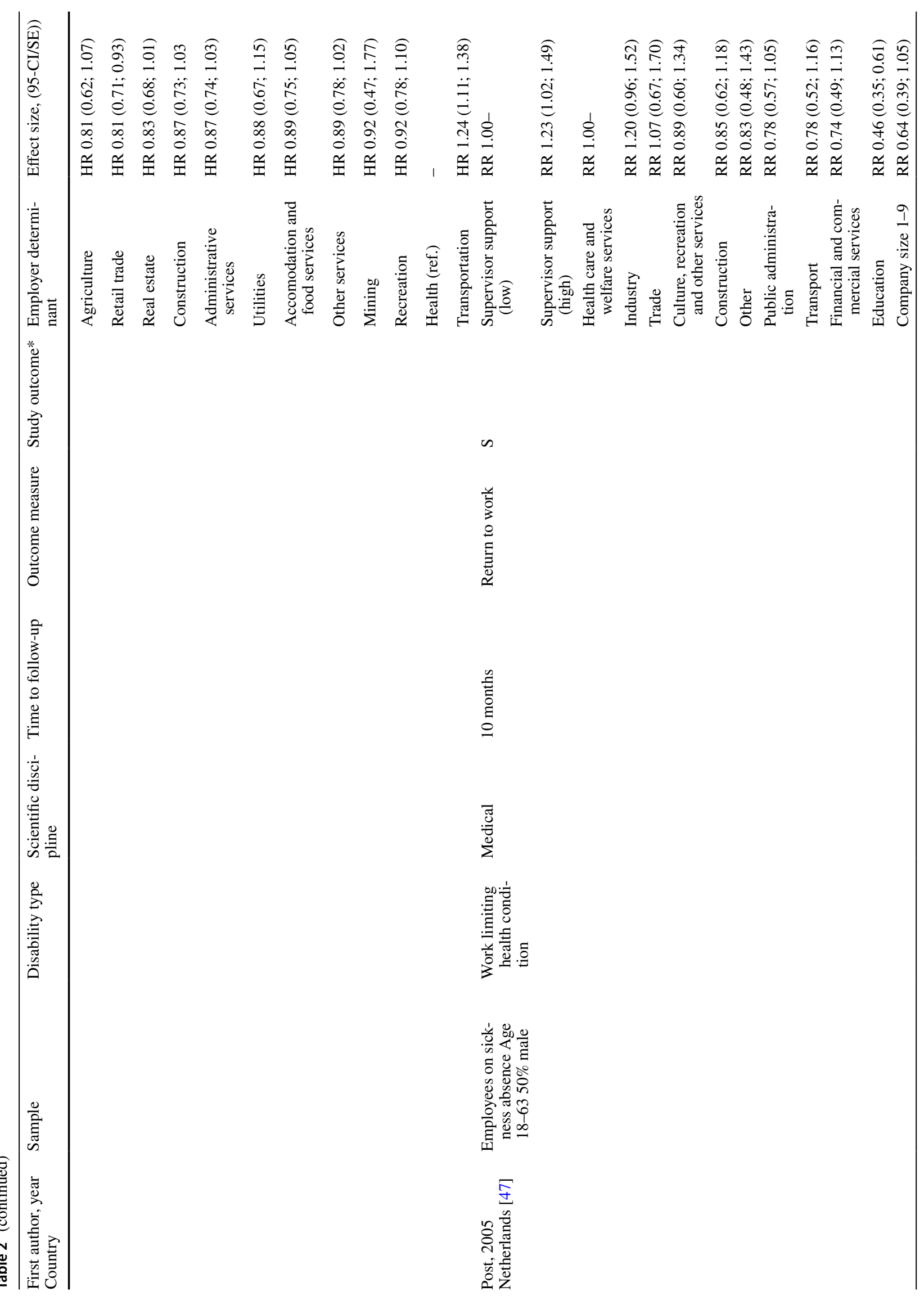




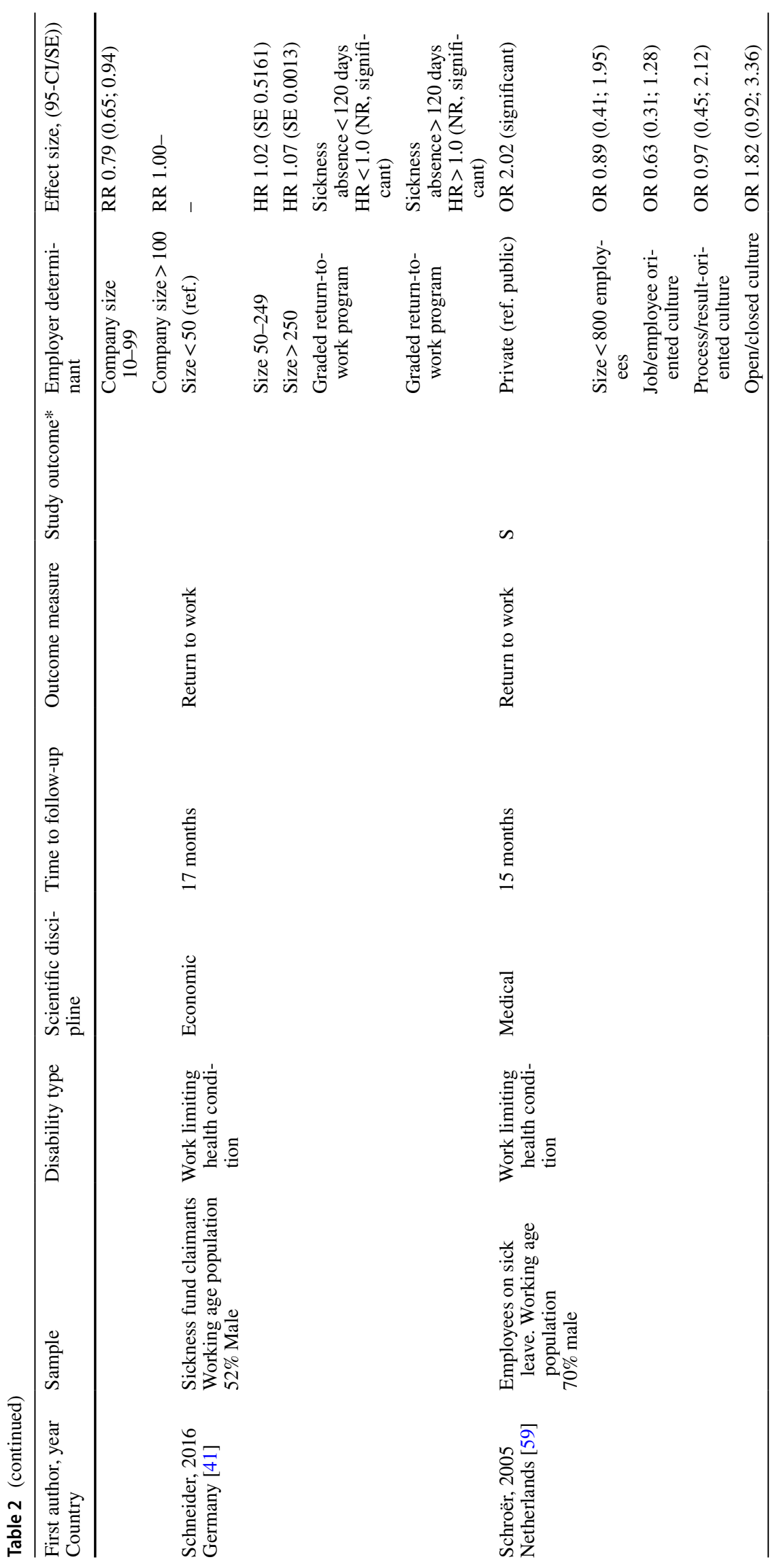




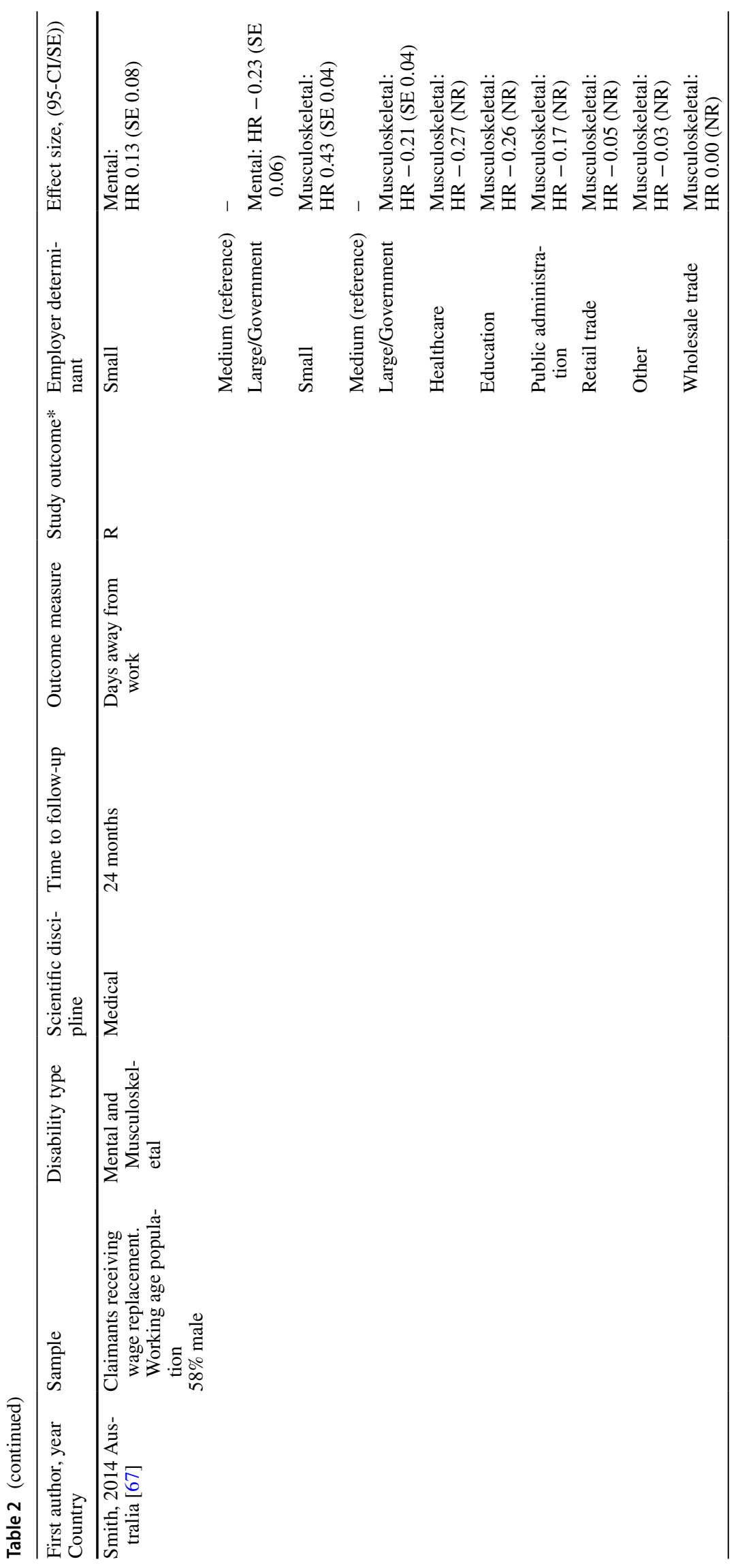




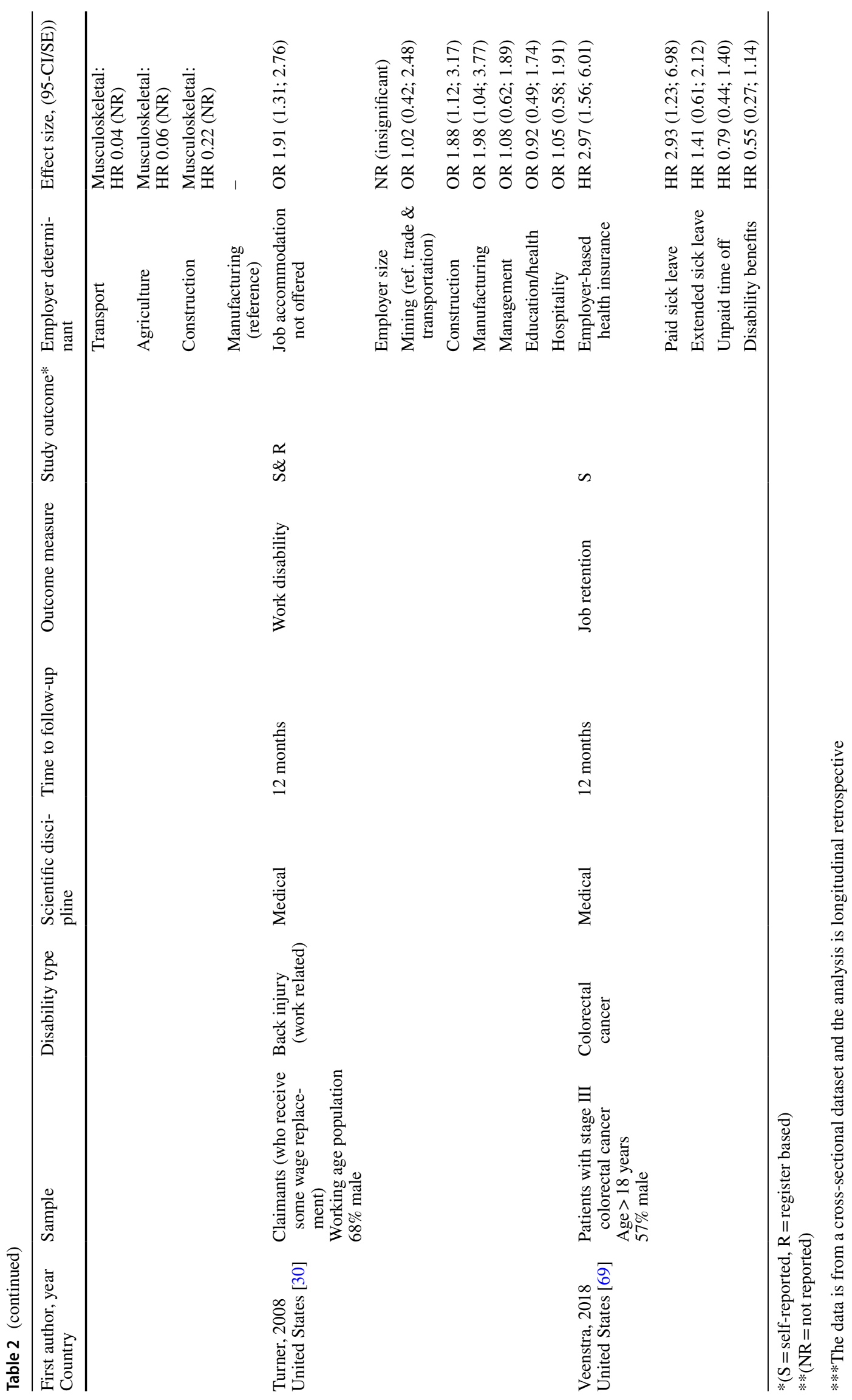




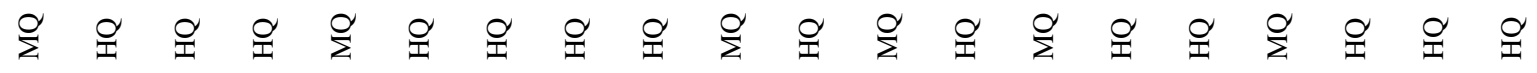

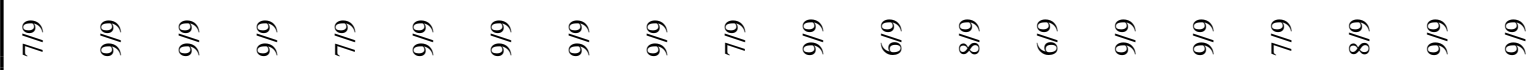
₹ $+$ $+$ $++$ $+$ $++$

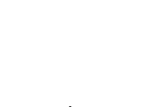
$+$

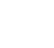




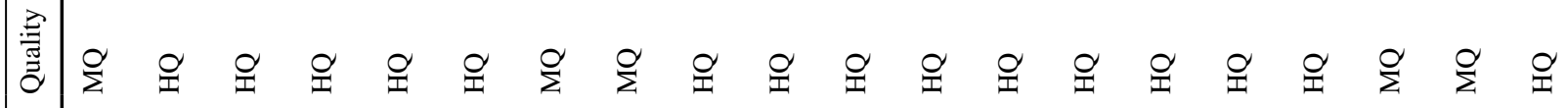

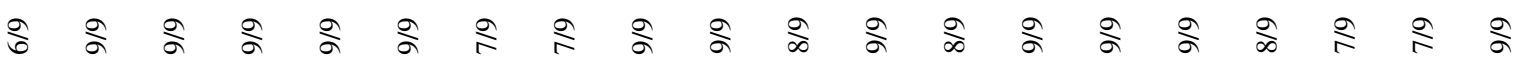

$a$

$\infty$

$r$

6 1 $1++++$

n

$+\int+\quad+\quad+\quad+\quad+$

n

ง

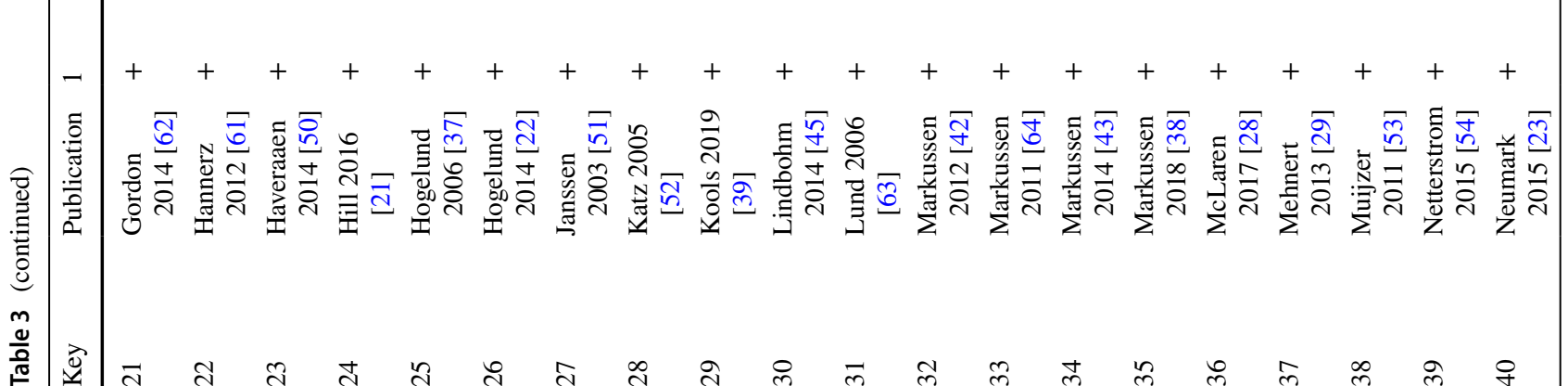




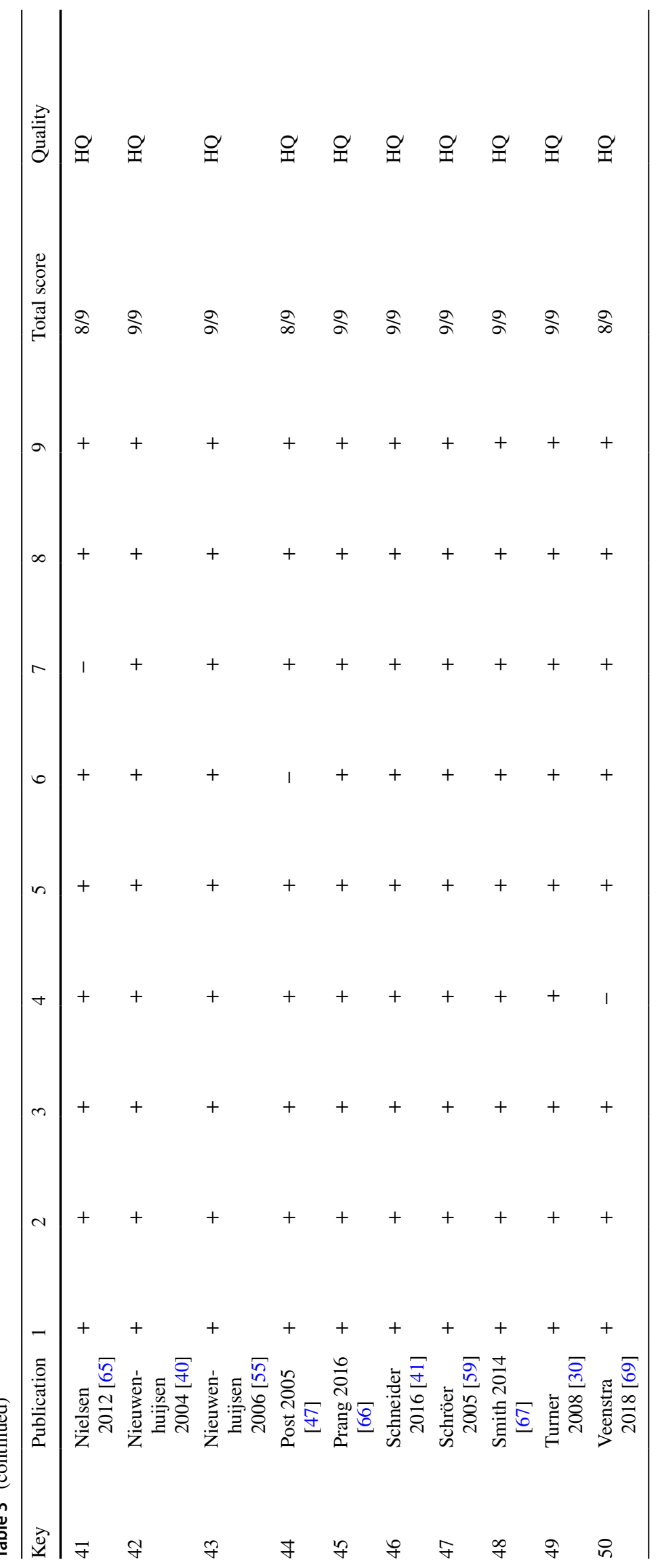


Table 4 Overview of evidence grading per determinant

\begin{tabular}{|c|c|c|c|c|c|c|c|c|}
\hline Domain & Determinants & $\begin{array}{l}\text { Work participation } \\
\text { outcome }\end{array}$ & Evidence & Nr. of studies & Ref. nr & Quality assessment & $\begin{array}{l}\text { Scientific disci- } \\
\text { pline }\end{array}$ & Disability type \\
\hline \multirow[t]{21}{*}{$\begin{array}{l}\text { Work accommo- } \\
\text { dation }\end{array}$} & \multirow[t]{3}{*}{$\begin{array}{l}\text { 1. Any accom- } \\
\text { modation }\end{array}$} & $\begin{array}{l}\text { Continued } \\
\text { employment }\end{array}$ & Strong + & 5 & [20-24] & $\operatorname{High}(\mathrm{n}=5)$ & $\begin{array}{l}\text { Economic }(n=4) \\
\text { Medical }(n=1)\end{array}$ & $\begin{array}{l}\text { Work-limiting health } \\
\text { condition }(n=3) \\
\text { Cancer }(n=2)\end{array}$ \\
\hline & & Return to work & Strong + & 5 & [25-29] & $\begin{array}{l}\text { High }(n=4) \\
\text { Medium }(n=1)\end{array}$ & $\begin{array}{l}\text { Economic }(n=2) \\
\text { Medical }(n=3)\end{array}$ & $\begin{array}{l}\text { Work-limiting health } \\
\text { condition }(n=2) \\
\text { Cancer }(n=2) \\
\text { Musculoskeletal }(n=1)\end{array}$ \\
\hline & & $\begin{array}{l}\text { Long-term dis- } \\
\text { ability }\end{array}$ & Moderate + & 3 & {$[21,30,31]$} & $\operatorname{High}(\mathrm{n}=3)$ & $\begin{array}{l}\text { Economic }(\mathrm{n}=2) \\
\text { Medical }(\mathrm{n}=1)\end{array}$ & $\begin{array}{l}\text { Work-limiting health } \\
\text { condition }(\mathrm{n}=2) \\
\text { Musculoskeletal }(\mathrm{n}=1)\end{array}$ \\
\hline & \multirow[t]{2}{*}{ 2. Work change } & $\begin{array}{l}\text { Continued } \\
\text { employment }\end{array}$ & Moderate + & 4 & {$[21-23,32]$} & $\operatorname{High}(n=4)$ & $\begin{array}{l}\text { Economic }(n=3) \\
\text { Medical }(n=1)\end{array}$ & $\begin{array}{l}\text { Work-limiting health } \\
\quad \text { condition }(n=2) \\
\text { Cancer }(n=1) \\
\text { Nervous }(n=1)\end{array}$ \\
\hline & & Return to work & Inconsistent & 3 & {$[28,33,35]$} & $\operatorname{High}(n=3)$ & $\begin{array}{l}\text { Economic }(n=1) \\
\text { Medical }(n=2)\end{array}$ & $\begin{array}{l}\text { Work-limiting health } \\
\text { condition }(n=1) \\
\text { Musculoskeletal }(n=1) \\
\text { Mental }(n=1)\end{array}$ \\
\hline & \multirow[t]{2}{*}{$\begin{array}{l}\text { 3. Employer } \\
\text { change }\end{array}$} & $\begin{array}{l}\text { Continued } \\
\text { employment }\end{array}$ & Inconsistent & 1 & {$[22,43]$} & $\operatorname{High}(\mathrm{n}=2)$ & Economic $(\mathrm{n}=2)$ & $\begin{array}{l}\text { Work-limiting health } \\
\text { condition }(\mathrm{n}=2)\end{array}$ \\
\hline & & $\begin{array}{l}\text { Long-term dis- } \\
\text { ability }\end{array}$ & Insufficient & 1 & [43] & $\operatorname{High}(\mathrm{n}=1)$ & Economic $(\mathrm{n}=1)$ & $\begin{array}{l}\text { Work-limiting health } \\
\text { condition }(\mathrm{n}=1)\end{array}$ \\
\hline & \multirow[t]{2}{*}{ 4. Time } & $\begin{array}{l}\text { Continued } \\
\text { employment }\end{array}$ & Moderate + & 3 & [21-23] & $\operatorname{High}(n=3)$ & Economic $(n=3)$ & $\begin{array}{l}\text { Work-limiting health } \\
\text { condition }(\mathrm{n}=2) \\
\text { Cancer }(\mathrm{n}=1)\end{array}$ \\
\hline & & Return to work & Strong + & 3 & {$[28,33,34]$} & $\begin{array}{l}\operatorname{High}(\mathrm{n}=2) \\
\text { Medium }(\mathrm{n}=1)\end{array}$ & $\begin{array}{l}\text { Medical }(\mathrm{n}=2) \\
\text { Economic }(\mathrm{n}=1)\end{array}$ & $\begin{array}{l}\text { Work-limiting health } \\
\text { condition }(n=1) \\
\text { Cancer }(n=1) \\
\text { Musculoskeletal }(n=1)\end{array}$ \\
\hline & \multirow[t]{2}{*}{$\begin{array}{l}\text { 5. Workplace } \\
\text { intervention }\end{array}$} & Return to work & Strong + & 6 & {$[26,33,35-38]$} & $\begin{array}{l}\operatorname{High}(\mathrm{n}=5) \\
\text { Medium }(\mathrm{n}=1)\end{array}$ & $\begin{array}{l}\text { Economic }(n=4) \\
\text { Medical }(n=2)\end{array}$ & $\begin{array}{l}\text { Work-limiting health } \\
\text { condition }(n=4) \\
\text { Musculoskeletal }(n=1) \\
\text { Mental }(n=1)\end{array}$ \\
\hline & & $\begin{array}{l}\text { Long-term dis- } \\
\text { ability }\end{array}$ & Insufficient & 1 & {$[35]$} & $\operatorname{High}(\mathrm{n}=1)$ & Medical $(n=1)$ & Mental $(\mathrm{n}=1)$ \\
\hline & \multirow[t]{3}{*}{$\begin{array}{l}\text { 6. Graded return to } \\
\text { work }\end{array}$} & $\begin{array}{l}\text { Continued } \\
\text { employment }\end{array}$ & Insufficient & 1 & {$[42]$} & $\operatorname{High}(\mathrm{n}=1)$ & Economic $(\mathrm{n}=1)$ & $\begin{array}{l}\text { Work-limiting health } \\
\text { condition }(\mathrm{n}=1)\end{array}$ \\
\hline & & Return to work & Weak + & 4 & [39-42] & $\operatorname{High}(n=4)$ & $\begin{array}{l}\text { Economic }(n=3) \\
\text { Medical }(n=1)\end{array}$ & $\begin{array}{l}\text { Work-limiting health } \\
\text { condition }(n=3) \\
\text { Mental }(n=1)\end{array}$ \\
\hline & & $\begin{array}{l}\text { Long-term dis- } \\
\text { ability }\end{array}$ & Insufficient & 1 & {$[42]$} & $\operatorname{High}(n=1)$ & Economic $(n=1)$ & $\begin{array}{l}\text { Work-limiting health } \\
\text { condition }(\mathrm{n}=1)\end{array}$ \\
\hline & \multirow[t]{2}{*}{$\begin{array}{l}\text { 7. Professional } \\
\text { assistance at } \\
\text { work }\end{array}$} & $\begin{array}{l}\text { Continued } \\
\text { employment }\end{array}$ & Insufficient & 1 & {$[23]$} & $\operatorname{High}(\mathrm{n}=1)$ & Economic $(\mathrm{n}=1)$ & Cancer $(\mathrm{n}=1)$ \\
\hline & & Return to work & Insufficient & 1 & {$[27]$} & $\operatorname{High}(\mathrm{n}=1)$ & Medical $(\mathrm{n}=1)$ & Musculoskeletal $(\mathrm{n}=1)$ \\
\hline & \multirow[t]{2}{*}{$\begin{array}{l}\text { 8. Professional } \\
\text { assistance } \\
\text { outside work }\end{array}$} & $\begin{array}{l}\text { Continued } \\
\text { employment }\end{array}$ & Insufficient & 1 & {$[23]$} & $\operatorname{High}(\mathrm{n}=1)$ & Economic $(\mathrm{n}=1)$ & Cancer $(\mathrm{n}=1)$ \\
\hline & & Return to work & Inconsistent & 3 & {$[26,27,40]$} & $\begin{array}{l}\operatorname{High}(\mathrm{n}=2) \\
\text { Medium }(\mathrm{n}=1)\end{array}$ & $\begin{array}{l}\text { Economic }(n=1) \\
\text { Medical }(n=2)\end{array}$ & $\begin{array}{l}\text { Work-limiting health } \\
\quad \text { condition }(n=1) \\
\text { Musculoskeletal }(n=1) \\
\text { Mental }(n=1)\end{array}$ \\
\hline & \multirow[t]{2}{*}{$\begin{array}{l}\text { 9. Equipment assis- } \\
\text { tance }\end{array}$} & $\begin{array}{l}\text { Continued } \\
\text { employment }\end{array}$ & Weak + & 3 & [21-23] & $\operatorname{High}(n=3)$ & Economic $(n=3)$ & $\begin{array}{l}\text { Work-limiting health } \\
\text { condition }(n=2) \\
\text { Cancer }(n=1)\end{array}$ \\
\hline & & Return to work & Strong + & 3 & {$[27,28,33]$} & $\operatorname{High}(n=3)$ & $\begin{array}{l}\text { Economic }(\mathrm{n}=1) \\
\text { Medical }(\mathrm{n}=2)\end{array}$ & $\begin{array}{l}\text { Work-limiting health } \\
\text { condition }(n=1) \\
\text { Musculoskeletal }(n=2)\end{array}$ \\
\hline & $\begin{array}{l}\text { 10. Employer } \\
\text { provided health/ } \\
\text { sick leave /dis- } \\
\text { ability insurance }\end{array}$ & $\begin{array}{l}\text { Continued } \\
\text { employment }\end{array}$ & Moderate + & 2 & {$[20,69]$} & $\operatorname{High}(\mathrm{n}=2)$ & Medical $(n=2)$ & Cancer $(\mathrm{n}=2)$ \\
\hline Social support & $\begin{array}{l}\text { 11. Supervisor } \\
\text { support }\end{array}$ & $\begin{array}{l}\text { Continued } \\
\text { employment }\end{array}$ & Weak + & 2 & {$[32,45]$} & $\operatorname{High}(\mathrm{n}=2)$ & Medical $(n=2)$ & $\begin{array}{l}\text { Cancer }(n=1) \\
\text { Nervous }(n=1)\end{array}$ \\
\hline
\end{tabular}


Table 4 (continued)

\begin{tabular}{|c|c|c|c|c|c|c|c|c|}
\hline Domain & Determinants & $\begin{array}{l}\text { Work participation } \\
\text { outcome }\end{array}$ & Evidence & Nr. of studies & Ref. nr & Quality assessment & $\begin{array}{l}\text { Scientific disci- } \\
\text { pline }\end{array}$ & Disability type \\
\hline & & Return to work & Moderate + & 14 & {$[40,44,46-55]$} & $\begin{array}{l}\text { High }(n=8) \\
\text { Medium }(n=6)\end{array}$ & Medical $(n=14)$ & $\begin{array}{l}\text { Work-limiting health } \\
\quad \text { condition }(n=3) \\
\text { Musculoskeletal }(n=2) \\
\text { Mental }(n=5) \\
\text { Diabetes }(n=3) \\
\text { Nervous }(n=1) \\
\text { Cancer }(n=1)\end{array}$ \\
\hline $\begin{array}{l}\text { Organizational } \\
\text { culture }\end{array}$ & $\begin{array}{l}\text { 12. Organizational } \\
\text { culture }\end{array}$ & Return to work & Weak + & 5 & {$[52,56-59]$} & $\begin{array}{l}\operatorname{High}(\mathrm{n}=2) \\
\text { Medium }(\mathrm{n}=3)\end{array}$ & Medical $(n=5)$ & $\begin{array}{l}\text { Work-limiting health } \\
\quad \text { condition }(n=1) \\
\text { Musculoskeletal }(n=1) \\
\text { Mental }(n=1) \\
\text { Circulatory }(n=1) \\
\text { Nervous }(n=1)\end{array}$ \\
\hline \multirow[t]{5}{*}{$\begin{array}{l}\text { Company charac- } \\
\text { teristics }\end{array}$} & 13. Company size & $\begin{array}{l}\text { Continued } \\
\text { employment/ }\end{array}$ & Inconsistent & 47 & {$[20,22,32,60]$} & $\operatorname{High}(n=4)$ & $\begin{array}{l}\text { Economic }(\mathrm{n}=1) \\
\text { Medical }(\mathrm{n}=3)\end{array}$ & $\begin{array}{l}\text { Work-limiting health } \\
\text { condition }(n=2) \\
\text { Cancer }(n=1) \\
\text { Nervous }(n=1)\end{array}$ \\
\hline & & Return to work & Inconsistent & 12 & $\begin{array}{c}{[34,41,47,52} \\
59,61-67]\end{array}$ & $\begin{array}{l}\operatorname{High}(n=9) \\
\text { Medium }(n=3)\end{array}$ & $\begin{array}{l}\text { Economic }(\mathrm{n}=2) \\
\text { Medical }(\mathrm{n}=10)\end{array}$ & $\begin{array}{l}\text { Work-limiting health } \\
\text { condition }(n=5) \\
\text { Musculoskeletal disorder } \\
\quad(n=1) \\
\text { Cancer }(n=2) \\
\text { Mental }(n=3) \\
\text { Nervous }(n=1) \\
\text { Circulatory }(n=1)\end{array}$ \\
\hline & & $\begin{array}{l}\text { Long-term dis- } \\
\text { ability }\end{array}$ & Insufficient & 1 & {$[30]$} & $\operatorname{High}(\mathrm{n}=1)$ & Medical $(\mathrm{n}=1)$ & $\begin{array}{l}\text { Musculoskeletal disorder } \\
\quad(\mathrm{n}=1)\end{array}$ \\
\hline & 14. Sector & $\begin{array}{l}\text { Continued } \\
\text { employment }\end{array}$ & Insufficient & 1 & {$[22]$} & $\operatorname{High}(\mathrm{n}=1)$ & Economic $(\mathrm{n}=1)$ & $\begin{array}{l}\text { Work-limiting health } \\
\text { condition }(\mathrm{n}=1)\end{array}$ \\
\hline & & Return to work & Inconsistent & 9 & $\begin{array}{c}{[37,47,59,} \\
63-68]\end{array}$ & $\operatorname{High}(\mathrm{n}=9)$ & $\begin{array}{l}\text { Economic }(\mathrm{n}=2) \\
\text { Medical }(\mathrm{n}=7)\end{array}$ & $\begin{array}{l}\text { Work-limiting health } \\
\text { condition }(n=5) \\
\text { Musculoskeletal }(n=1) \\
\text { Mental }(n=4)\end{array}$ \\
\hline
\end{tabular}

disability, this concerns the determinants employer change [43], workplace interventions [35], graded return to work [42].

\section{Social Support}

Social support, includes measures of the relationship between the supervisor and the worker, measures of supervisor support and measures relating to the presence of conflicts between supervisor and worker. Weak evidence was found for a positive association with continued employment [32, 45]. For return to work moderate evidence was found for this association [40, 44, 46-55]. No studies were found for long-term disability.

\section{Organizational Culture}

Determinants related to organizational culture, like injustice, open versus closed culture, less supportive policies and practices were only studied in relation to return to work. The overall evidence for these determinants was weak [52, 56-59].

\section{Company Characteristics}

Two company characteristics identified in the included studies of interest were company size and sector. Inconsistent evidence was found for the associations between company size and continued employment $[20,22,32,60]$ and return to work [34, 41, 47, 52, 59, 61-67]. Insufficient evidence was found for long-term disability [30]. When comparing the public and private sectors, insufficient evidence was found for the association between the sector of employment and continued employment [22]. Furthermore, inconsistent evidence was found for the association between sector of employment and return to work [37, 47, 59, 63-68]. No studies were found for long-term disability with regard to sector.

\section{Discussion}

In this systematic literature review, we explored the determinants at employer level associated with continued employment, return to work, and long-term work disability of workers with disabilities. Our findings indicate that organizational efforts on both supervisor level (i.e., work accommodations, support) and higher organizational levels (i.e., culture, policy), as well as company characteristics (i.e., 
sector, company size) can influence these work outcomes. At supervisor level, strong evidence was found for work accommodations. In addition, weak to moderate evidence was found for social support. Evidence for employer efforts at higher organizational levels was weak. Evidence for an association between company characteristics and continued employment, return to work and long-term disability was inconsistent.

\section{Supervisor Level: Work Accommodations}

At supervisor level, our findings indicate that providing work accommodations is positively associated with continued employment and return to work, and negatively with long-term disability. The strength of evidence differed between work accommodation categories and the three work outcomes. We found strong evidence for the benefits of work accommodations concerning adaptations to work schedules for return to work, such as having the option to choose for flexible working hours [34] and to reduce working hours [28, 33]. We also found strong evidence for work accommodations concerning workplace adaptations, like the provision of a laptop computer that allowed workers to work from home [28], and changes in furniture at the office or workstation [27, 28, 33]. Moreover, we found strong evidence for work accommodations concerning interventions that aim to provide workers with additional support and guidance associated with return to work [26, 28, 33, 35-38]. These interventions focused on providing a workplace-oriented rehabilitation program like vocational work training or educational training, but also on providing occupational health services and case management interviews. We found moderate evidence for work accommodations regarding employer-provided changes in work in relation to continued employment [21-23, 32] which consisted of modifications to either work activities and duties [21, 23, 32] or the offer of a new job in the same company [22]. Additionally, we found moderate evidence for an association between employer-provided disability insurances [20,69] and continued employment. For long-term work disability, we found insufficient evidence for work accommodations, which can be explained by the low number of articles available for this outcome.

The finding that offering work accommodations facilitates work participation is in line with previous reviews that reported on the evidence for adaptations to work schedules, providing equipment and modifications to work activities [6, $10,16,70-73]$. However, most reviews studied work accommodations in relation to returning to work after sickness absence, but did not consider associations with continued employment and long-term work disability. For example, we found evidence that modifications to work activities are not only helpful for workers returning to work [73], but are also important in the context of staying employed after the onset of work disability. Our findings are consistent across different causes of work disabilities.

\section{Supervisor Level: Social Support}

We found moderate evidence that social support from supervisors was related to return to work. Social support was operationalized as supervisor support as perceived by the worker [49-52, 54], a positive relation between supervisor and worker [53] and the supervisors' communication with and response to workers $[40,46]$. We found weak evidence for an association of social support from supervisors with continued employment $[32,45]$, which may be explained by the low number of included studies on this outcome. There were no articles included with long-term work disability as outcome.

The finding that social support facilitates work participation is consistent with several reviews [74-76] which found moderate-to-strong evidence for a positive relation between supervisor support and a shorter duration of sick leave, and reduction of workplace disability. However, two previous reviews on return to work, found no evidence for a positive relation of social support with return to work (yes/no) [77, 78]. This may be explained by the lower number of studies included in those return to work reviews compared to our study, as a consequence of these studies focusing on a specific disease group (e.g. cardiovascular disease and mental health). Compared with these two prior reviews, our review adds evidence concerning particular relational aspects of social support that are relevant for work participation of workers with all kind of work disabilities.

\section{Organizational Level: Culture}

At organizational level, we found weak evidence for a positive association between organizational culture and return to work. Organizational culture includes a variety of determinants regarding the nature of the organizational culture (e.g. a people oriented culture, process or result oriented culture, open or closed culture, reward system, justice within an organization) [57-59], as well as determinants regarding organizational policies and practices (e.g. disability management programs and ergonomic policies) [52, 56]. No articles were included with either continued employment or longterm work disability as outcome.

There are some reviews on policies and practices (e.g. workplace disability management programs) that found insufficient evidence for an association with return to work $[79,80]$. These reviews concluded that conclusions could not be made due to lack of evidence and high risk of bias in their included studies. Overall, more research on this topic is needed, as only a few studies could be included in our review. Moreover, there is a large variety in measurement 
of organizational culture across studies, as culture seems difficult to capture in questionnaires [81].

\section{Comparison of Findings Between Types of Diseases}

In this systematic review, we included studies on workers with a broad range of disease groups. Because we included studies with different diseases we could provide an overview of prognostic factors that are relevant across different diseases, without specifically studying for differences between the disease groups. In almost half of these studies, the study population was defined as workers with work-limiting health conditions, i.e. all kinds of disability types were included and no distinction was made between the types of diseases. These studies were often found in the economic database. In contrast, studies from the field of medicine, occupational health and psychology often focused on a specific disease group, and included workers with a specific disability type, like mental health $[35,40,48,53,58,65,66,68]$, musculoskeletal disorders [27, 33, 46, 56, 67], and cancer [20, 25, $29,34,44,45,62]$.

Comparison of the studies showed that studies including workers with work-limiting health conditions mainly focused on the employer-domains work accommodations and company characteristics. For the disease-specific studies, we found that studies on mental health mostly focused on social support and company characteristics, whereas studies on musculoskeletal disorders and cancer mainly focused on work accommodations and company characteristics.

Comparison of the evidence showed that all studies including workers with work-limiting health conditions found positive evidence for an association between social support and work [47, 50, 51], whereas seven out of eleven studies on specific disease groups, like mental health, musculoskeletal disorders and cancer, found insignificant evidence for this association [32, 40, 44-46, 48, 49, 52-55]. We did not find any differences in evidence for specific work accommodations between the disease groups, nor between the specific disease groups in relation to the outcomes. This is in line with a previous study on supervisor competencies for supporting return to work following absence due to a mental health condition or a musculoskeletal disorder that showed that supervisor competencies relevant for return to work did not differ between workers with different chronic diseases [82]. Due to the low number of included studies on organizational culture, it was not possible to further analyze these findings. For the domain company characteristics, most studies found insignificant or even inconsistent evidence. For this reason, differences between generic and disease-specific studies and between disease groups were not studied.

\section{Strengths and Limitations}

A strength of this review is that we included determinants of work participation at both supervisor level and organizational level. This provides a comprehensive overview of relevant employer determinants on different employer levels, in which context both the supervisor and organizational level plays a role.

Another strength of this review is that we only included longitudinal quantitative studies, which allowed us to summarize the evidence of the associations between the employer determinants and the work outcomes. However, the decision to exclude studies with a qualitative design entails that we excluded studies that could have provided more in-depth information about determinants like organizational culture and policies and practices.

Moreover, a strength of this review is the interdisciplinary perspective. Every included scientific field had their own contribution to our research topic. The economic studies primarily focused on continued employment, while medical and occupational health studies focused more on the return to work outcome. In the economic literature, the scope of studies was mostly on work accommodations and company characteristics, whereas the medical field focused on all the different employer domains. Furthermore, the economic studies mostly included data related to workers with work-limiting disabilities, whereas the medical, psychological and occupational health studies generally used data related to workers of specific disease groups. The inclusion of studies from these different fields enabled us to compare different outcome measures. The large consistency of the findings across the different outcome measures, makes us more confident about the strength of the presented evidence in our review, but also illustrate the added value of our interdisciplinary approach.

This study also has some limitations. In the field of economics it is common to publish working papers of submitted manuscripts because of the relatively long publishing process. In consequence of the decision not to include working papers we might have missed relevant recent papers from the economic perspective. Furthermore, we excluded studies in languages other than English and all included studies were from high-income countries. Consequently, we might have missed some useful studies from non-western countries, which may restrict the generalizability of the findings.

\section{Implications for Practice and Future Research}

This review supports the assumption that the employer has a role in work participation of workers with disabilities. In particular, various work accommodations and supervisor support were found to be important for return to work and continued employment. However, for some work accommodations, like change of employer, job change, and professional 
assistance at- and outside of work, more research is needed on the impact on continued employment, return to work and long-term disability. Additionally, although supervisor support is a consistent determinant across the studies, further quantitative research is needed on supervisor support, which may include other aspects of social support, like instrumental or emotional support. Future research should therefore focus on the association between work outcomes and aspects of social support that have been found to be important in other studies. In this study, we cannot draw strong conclusions on the influence of culture and policies and practices due to the limited number of studies on organizational culture and organizational policies and practices, and the inconsistent measurement of organizational culture. Similarly, we found inconsistent evidence for company characteristics, which might be due to different classifications of company size and sector of employment. As organizational culture, policies and practices, and company characteristics could be important facilitators for employer support, further research is needed on the influence of these higher organizational levels on continued employment, return to work and long-term disability. Especially, more research is needed on how to measure the aspects of organizational culture that may be relevant for continued employment, return to work and long-term disability.

\section{Conclusion}

This systematic literature review including studies from the economic, medical, psychological and occupational health field shows that employer support enables workers with disabilities to continue employment and return to work or reduce the likelihood of long-term work disability. Employer support entails organizational efforts on supervisor level and organizational level, as well as the role of company characteristics. This review especially shows positive evidence for the facilitation of work accommodations and for support of supervisors in relation with the above mentioned work outcomes. The evidence seems to be valid across studies that focused on specific and generic disease groups. Despite the weak evidence for organizational culture and inconsistent evidence for company size and sector of employment, our review indicates the importance of employer efforts on different organizational levels for preventing early labor market exit of workers with poor health. We found consistent evidence for a positive effect of efforts on supervisor level on the work participation outcomes. The role of organizational culture is less clear due to a weak level of evidence. However, as organizational culture is found to be important in qualitative studies, more research is needed on factors related to this concept. In this context, it is important for future longitudinal studies to achieve more consensus on the measurement of social support and organizational culture and policies.
Supplementary Information The online version contains supplementary material available at https://doi.org/10.1007/s10926-021-09978-3.

Acknowledgements The authors would like to thank Truus van Ittersum, for her contribution in the search strategy.

Funding This work was supported by Instituut Gak, Grant Number 2018-933.

\section{Declarations}

Conflict of interest All authors declare that they have no conflict of interest.

Open Access This article is licensed under a Creative Commons Attribution 4.0 International License, which permits use, sharing, adaptation, distribution and reproduction in any medium or format, as long as you give appropriate credit to the original author(s) and the source, provide a link to the Creative Commons licence, and indicate if changes were made. The images or other third party material in this article are included in the article's Creative Commons licence, unless indicated otherwise in a credit line to the material. If material is not included in the article's Creative Commons licence and your intended use is not permitted by statutory regulation or exceeds the permitted use, you will need to obtain permission directly from the copyright holder. To view a copy of this licence, visit http://creativecommons.org/licenses/by/4.0/.

\section{References}

1. OECD. Sickness, Disability and Work - A Synthesis of Findings across OECD Countries. 2010. 1-556 pp.

2. Burkhauser R, Daly M, Ziebarth N. Protecting working-age people with disabilities: experiences of four industrialized nations. J Labour Mark Res. 2016;49:367-386.

3. Clayton S, Barr B, Nylen L, Burstrom B, Thielen K, Diderichsen $\mathrm{F}$, et al. Effectiveness of return-to-work interventions for disabled people: a systematic review of government initiatives focused on changing the behaviour of employers. Eur J Public Health. 2012;22(3):434-439.

4. Böheim R, Leoni T. Sickness and disability policies: reform paths in OECD countries between 1990 and 2014. Int J Soc Welf. 2018;27(2):168-185.

5. Organization WH. International Classification of Functioning, Disability and Health World Health Organization Geneva ICF ii WHO Library Cataloguing-in-Publication Data International classification of functioning, disability and health: ICF2001.

6. Franche R-L, Baril R, Shaw W, Nicholas M, Loisel P. Workplace-based return-to-work interventions: optimizing the role of stakeholders in implementation and research. J Occup Rehabil. 2005;15(4):525-42.

7. Shaw WS, Main CJ, Pransky G, Nicholas MK, Anema JR, Linton SJ. Employer policies and practices to manage and prevent disability: foreward to the special issue. J Occup Rehabil. 2016;26(4):394-8.

8. MacDonald-Wilson KL, Fabian ES, Dong S. Best practices in developing reasonable accommodations in the workplace: findings based on the research literature. Rehabil Prof. 2008;16(4):221-32.

9. De Croon EM, Sluiter JK, Nijssen TF, Dijkmans AC, Lankhorst GJ. Predictive factors of work disability in rheumatoid arthritis: a systematic literature review. Ann Rheum Dis. 2004;63:1362-7.

10. Nazarov S, Manuwald U, Leonardi M, Silvaggi F, Foucaud J, Lamore K, et al. Chronic diseases and employment: which 
interventions support the maintenance of work and return to work among workers with chronic illnesses? A systematic review. Int J Environ Res Public Health. 2019;16(10): 1864.

11. Detaille S, Heerkens Y, Engels J, van der Gulden J, van Dijk F. Common prognostic factors of work disability among employees with a chronic somatic disease: a systematic review of cohort studies. Scand J Work Environ Health. 2009;35(4):261-81.

12. Williams RM, Westmorland MG, Lin CA, Schmuck G, Creen $M$. Effectiveness of workplace rehabilitation interventions in the treatment of work-related low back pain: a systematic review. Disabil Rehabil. 2007;29(8):607-24.

13. Lagerveld SE, Bü U, Franche RL, Van Dijk FJH, Vlasveld C, Van Der Feltz-Cornelis CM, et al. Factors associated with work participation and work functioning in depressed workers: a systematic review. J Occup Rehabil. 2010;20:275-92.

14. Islam T, Dahlui M, Majid H, Nahar A, Mohd Taib N, Su T. Factors associated with return to work of breast cancer survivors: a systematic review. BMC Public Health. 2014;14(3):S8.

15. Greidanus MA, de Boer AGEM, de Rijk AE, Tiedtke CM, Dierckx de Casterle B, Frings-Dresen MHW, et al. Perceived employerrelated barriers and facilitators for work participation of cancer survivors: a systematic review of employers' and survivors' perspectives. Psychooncology. 2018;27(3):725-33.

16. Padkapayeva K, Posen A, Yazdani A, Buettgen A, Mahood Q, Tompa E. Workplace accommodations for persons with physical disabilities: evidence synthesis of the peer-reviewed literature. Disabil Rehabil. 2017;39:2134-47.

17. Ouzzani M, Hammady H, Fedorowicz Z, Elmagarmid A. Rayyan-a web and mobile app for systematic reviews. Syst Rev. 2016;5(1):1-10.

18. Scharn M, Sewdas R, Boot CRL, Huisman M, Lindeboom M, Van Der Beek AJ. Domains and determinants of retirement timing: a systematic review of longitudinal studies. BMC Public Health. 2018;18(1): 1083 .

19. Hayden JA, Van Der Windt DA, Cartwright JL, Cô P, Bombardier C. Assessing bias in studies of prognostic factors. Ann Intern Med. 2013;158(4):280-6.

20. Blinder V, Eberle C, Patil S, Gany FM, Bradley CJ. Women with breast cancer who work for accommodating employers more likely to retain jobs after treatment. Health Aff (Millwood). 2017;36(2):274-81.

21. Hill MJ, Maestas N, Mullen KJ. Employer accommodation and labor supply of disabled workers. Labour Econ. 2016;41:291-303.

22. Høgelund J, Holm A. Worker adaptation and workplace accommodations after the onset of an illness. IZA J Labor Policy. 2014;3(1): 17.

23. Neumark D, Bradley CJ, Henry M, Dahman B. Work continuation while treated for breast cancer. ILR Rev. 2015;68(4):916-54.

24. Burkhauser RV, Butler JS, Kim YW. The importance of employer accommodation on the job duration of workers with disabilities: a hazard model approach. Labour Econ. 1995;2(2):109-30.

25. Bouknight RR, Bradley CJ, Luo Z, Bouknight RR. Correlates of return to work for breast cancer survivors community-clinical collaboration view project correlates of return to work for breast cancer survivors. Artic J Clin Oncol. 2006;24:345-53.

26. Everhardt TP, de Jong PR. Return to work after long term sickness. Economist (Leiden). 2011;159(3):361-80.

27. Franche R-L, Severin CN, Hogg-Johnson S, Côté P, Vidmar M, Lee $\mathrm{H}$. The impact of early workplace-based return-to-work strategies on work absence duration: a 6-month longitudinal study following an occupational musculoskeletal injury. J Occup Environ Med. 2007;49(9):960-74.

28. McLaren CF, Reville RT, Seabury SA. how effective are employer return to work programs? Int Rev Law Econ. 2017;52:58-73.
29. Mehnert A. Predictors of employment among cancer survivors after medical rehabilitation-a prospective study. Scand J Work Environ Health. 2013;39(1):76-87.

30. Turner JA, Franklin G, Fulton-Kehoe D, Sheppard L, Stover B, Wu R, et al. ISSLS Prize Winner: early predictors of chronic work disability: a prospective, population-based study of workers with back injuries. Spine (Phila Pa 1976). 2008;33(25):2809-18.

31. Burkhauser RV, Butler JS, Kim Y-W, Weathers RR. The importance of accommodation on the timing of disability insurance applications: results from the survey of disability and work and the health and retirement study. J Hum Resour. 1999;34(3):589-611.

32. Faucett J, Blanc P. The impact of carpal tunnel syndrome on work status: implications of job characteristics for staying on the job. $\mathrm{J}$ Occup Rehabil. 2000;10(1):55-69.

33. Anema JR, Schellart AJM, Cassidy JD, Loisel P, Veerman TJ, van der Beek AJ. Can cross country differences in return-to-work after chronic occupational back pain be explained? An Exploratory analysis on disability policies in a six country cohort study. J Occup Rehabil. 2009;19(4):419-26.

34. Cooper AF, Hankins M, Rixon L, Eaton E, Grunfeld EA. Distinct work-related, clinical and psychological factors predict return to work following treatment in four different cancer types. Psychooncology. 2013;22(3):659-67.

35. Bryngelson A, Mittendorfer-Rutz E, Jensen I, Lundberg U, Asberg M, Nygren A. Self-reported treatment, workplace-oriented rehabilitation, change of occupation and subsequent sickness absence and disability pension among employees long. BMJ Open. 2012;2(6):1-9.

36. Frölich M, Heshmati A, Lechner M. A microeconometric evaluation of rehabilitation of long-term sickness in Sweden. J Appl Econom. 2004;19(3):375-96.

37. Hogelund J, Holm A. Case management interviews and the return to work of disabled employees. J Health Econ. 2006;25(3):500-19.

38. Markussen S, Røed K, Schreiner RC. Can compulsory dialogues nudge sick-listed workers back to work? Econ J. 2018;128(610):1276-303.

39. Kools L, Koning P. Graded return-to-work as a stepping stone to full work resumption. J Health Econ. 2019;65:189-209.

40. Nieuwenhuijsen K, Verbeek JHAM, de Boer AGEM, Blonk RWB, van Dijk FJH. Supervisory behaviour as a predictor of return to work in employees absent from work due to mental health problems. Occup Environ Med. 2004;61(10):817-23.

41. Schneider U, Linder R, Verheyen F. Long-term sick leave and the impact of a graded return-to-work program: evidence from Germany. Eur J Health Econ. 2016;17(5):629-43.

42. Markussen S, Mykletun A, Røed K. The case for presenteeismevidence from Norway's sickness insurance program. J Public Econ. 2012;96(11-12):959-72.

43. Markussen $S$, Røed K. The impacts of vocational rehabilitation. Labour Econ. 2014;31:1-13.

44. Dorland HF, Abma FI, Van Zon SKR, Stewart RE, Amick BC, Ranchor AV, et al. Fatigue and depressive symptoms improve but remain negatively related to work functioning over 18 months after return to work in cancer patients. J Cancer Surv. 2018;12(3):371-8.

45. Lindbohm M-L, Kuosma E, Taskila T, Hietanen P, Carlsen K, Gudbergsson S, et al. Early retirement and non-employment after breast cancer. Psychooncology. 2014;23(6):634-41.

46. Boot CRL, Hogg-Johnson S, Bültmann U, Amick BC, van der Beek AJ. Differences in predictors for return to work following musculoskeletal injury between workers with and without somatic comorbidities. Int Arch Occup Environ Health. 2014;87(8):871-9.

47. Post M, Krol B, Groothoff JW. Work-related determinants of return to work of employees on long-term sickness absence. Disabil Rehabil. 2005;27(9):481-8. 
48. de Vries G, Koeter M, Nieuwenhuijsen K, Hees HL, Schene AH. Predictors of impaired work functioning in employees with major depression in remission. J Affect Disord. 2015;185:180-7.

49. Ervasti J, Kivimäki M, Dray-Spira R, Head J, Goldberg M, Pentti $\mathrm{J}$, et al. Psychosocial factors associated with work disability in men and women with diabetes: a pooled analysis of three occupational cohort studies. Diabet Med. 2016;33(2):208-17.

50. Haveraaen L, Skarpaas L, Berg J, Aas R. Do psychological job demands, decision control and social support predictreturn to work three months after a return-to-work (RTW) programme? The rapid-RTW. Work. 2016;53(1):61-71.

51. Janssen N, Van den Heuvel W, Beurkens A, Nijhuis F, Schröer C, van Eijk J. The demand-control-support model as a predictor of return to work. Int J Rehabil Res. 2003;26(1):1-9.

52. Katz JN, Amick BC, Keller R, Fossel AH, Ossman J, Soucie V, et al. Determinants of work absence following surgery for carpal tunnel syndrome. Am J Ind Med. 2005;47(2):120-30.

53. Muijzer A, Groothoff JW, Geertzen JHB, Brouwer S. Influence of efforts of employer and employee on return-to-work process and outcomes. J Occup Rehabil. 2011;21(4):513-9.

54. Netterstrøm B, Eller NH, Borritz M. Prognostic factors of returning to work after sick leave due to work-related common mental disorders: a one- and three-year follow-up study. Biomed Res Int. 2015;2015:1-7.

55. Nieuwenhuijsen K, Verbeek J, de Boer A, Blonk R, van Dijk F. Predicting the duration of sickness absence for patients with common mental disorders in occupational health care. Scand J Work Environ Health. 2006;32(1):67-74.

56. Amick BC, Lee H, Hogg-Johnson S, Katz JN, Brouwer S, Franche $\mathrm{R}-\mathrm{L}$, et al. How do organizational policies and practices affect return to work and work role functioning following a musculoskeletal injury? J Occup Rehabil. 2017;27(3):393-404.

57. Biering K, Lund T, Andersen JH, Hjollund NH. Effect of psychosocial work environment on sickness absence among patients treated for ischemic heart disease. J Occup Rehabil. 2015;25(4):776-82.

58. Ekberg K, Wåhlin C, Persson J, Bernfort L, Öberg B. Early and late return to work after sick leave: predictors in a cohort of sicklisted individuals with common mental disorders. J Occup Rehabil. 2015;25(3):627-37.

59. Schröer CAP, Janssen M, Van Amelsvoort LGPM, Bosma H, Swaen GMH, Nijhuis FJN, et al. Organizational characteristics as predictors of work disability: a prospective study among sick employees of for-profit and not-for-profit organizations. J Occup Rehabil. 2005;15(3):435-45.

60. Daly MC, Bound J. Worker adaptation and employer accommodation following the onset of a health impairment. J Gerontol. 1996;5(2):53-9.

61. Hannerz H, Ferm L, Poulsen OM, Pedersen BH, Andersen LL. Enterprise size and return to work after stroke. J Occup Rehabil. 2012;22(4):456-61.

62. Gordon LG, Beesley VL, Lynch BM, Mihala G, McGrath C, Graves N, et al. The return to work experiences of middle-aged Australian workers diagnosed with colorectal cancer: a matched cohort study. BMC Public Health. 2014;14(1):963.

63. Lund T, Labriola M. Return to work among sickness-absent Danish employees: prospective results from the Danish work environment cohort study/national register on social. Int J Rehabil Res. 2006;29(3):229-35.

64. Markussen S, Røed K, Røgeberg OJ, Gaure S. The anatomy of absenteeism. J Health Econ. 2011;30(2):277-92.

65. Nielsen MBD, Bultmann U, Madsen IEH, Martin M, Christensen U, Diderichsen F, et al. Health, work, and personal-related predictors of time to return to work among employees with mental health problems. Disabil Rehabil. 2012;34(15):1311-6.
66. Prang K, Bohensky M, Smith P, Collie A. Return to work outcomes for workers with mental health conditions: a retrospective cohort study. Injury. 2016;47(1):257-65.

67. Smith PM, Black O, Keegel T, Collie A. Are the predictors of work absence following a work-related injury similar for musculoskeletal and mental health claims? J Occup Rehabil. 2014;24(1):79-88.

68. Engström L-G, Janson S. Stress-related sickness absence and return to labour market in Sweden. Disabil Rehabil. 2007;29(5):411-6.

69. Veenstra CM, Abrahamse P, Wagner TH, Hawley ST, Banerjee M, Morris AM. Employment benefits and job retention: evidence among patients with colorectal cancer. Cancer Med. 2018;7(3):736-45.

70. Varekamp I, Verbeek JHAM, van Dijk FJH. How can we help employees with chronic diseases to stay at work? A review of interventions aimed at job retention and based on an empowerment perspective. Int Arch Occup Environ Health. 2006;80(2):87-97.

71. Nevala N, Pehkonen I, Koskela I, Ruusuvuori J, Anttila H. Workplace accommodation among persons with disabilities: a systematic review of its effectiveness and barriers or facilitators. J Occup Rehabil. 2015;25(2):432-48.

72. Noordik E, Nieuwenhuijsen K, Varekamp I, van der Klink JJ, van Dijk FJ. Exploring the return-to-work process for workers partially returned to work and partially on long-term sick leave due to common mental disorders: a qualitative study. Disabil Rehabil. 2011;33(17-18):1625-35.

73. Krause N, Dasinger L, Neuhauser F. Modified work and return to work: a review of the literature. J Occup Rehabil. 1998;8(2):113-39.

74. Steenstra IA, Verbeek JH, Heymans MW, Steenstra A. Prognostic factors for duration of sick leave in patients sick listed with acute low back pain: a systematic review of the literature. Occup Environ Med. 2005;62(12):851-60.

75. Nieuwenhuijsen K, Bruinvels D, Frings-Dresen M. Psychosocial work environment and stress-related disorders, a systematic review. Occup Med (Chic Ill). 2010;60(4):277-86.

76. White M, Wagner S, Schultz IZ, Murray E, Bradley SM, Hsu V, et al. Modifiable workplace risk factors contributing to workplace absence across health conditions: a stakeholder-centered best-evidence synthesis of systematic reviews. Work. 2013;45:475-92.

77. Blank L, Peters J, Pickvance S, Wilford J, MacDonald E. A systematic review of the factors which predict return to work for people suffering episodes of poor mental health. J Occup Rehabil. 2008;18(1):27-34.

78. Wozniak MA, Kittner SJ. Return to work after ischemic stroke: a methodological review. Neuroepidemiology. 2002;21:159-66.

79. Gensby U, Labriola M, Irvin E, Amick BC, Lund T. A classification of components of workplace disability management programs: results from a systematic review. J Occup Rehabil. 2014;24:220-41.

80. Steenstra IA, Munhall C, Irvin E, Oranye N, Passmore S, Van Eerd D, et al. Systematic review of prognostic factors for return to work in workers with sub acute and chronic low back pain. $\mathrm{J}$ Occup Rehabil. 2016;27:369-81.

81. Lundberg CC. Designing organizational culture courses: fundamental considerations. J Manag Educ. 1996;20(1):11-22.

82. Johnston V, Way K, Long MH, Wyatt M, Gibson L, Shaw WS. Supervisor competencies for supporting return to work: a mixedmethods study. J Occup Rehabil. 2015;25(1):3-17.

Publisher's Note Springer Nature remains neutral with regard to jurisdictional claims in published maps and institutional affiliations. 